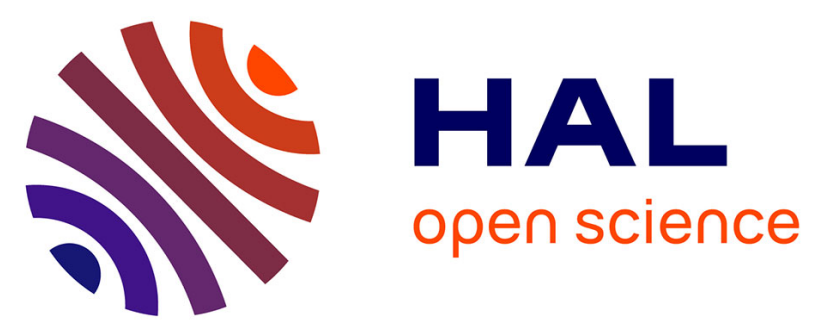

\title{
Fabrication of Oriented n-Type Thermoelectric Polymers by Polarity Switching in a DPP-Based Donor-Acceptor Copolymer Doped with FeCl 3
}

Huiyan Zeng, Mohammed Mohammed, Viktoriia Untilova, Olivier Boyron, Nicolas Berton, Patrice Limelette, Bruno Schmaltz, Martin Brinkmann

\section{To cite this version:}

Huiyan Zeng, Mohammed Mohammed, Viktoriia Untilova, Olivier Boyron, Nicolas Berton, et al.. Fabrication of Oriented n-Type Thermoelectric Polymers by Polarity Switching in a DPP-Based DonorAcceptor Copolymer Doped with FeCl 3. Advanced Electronic Materials, 2021, 7 (5), pp.2000880. 10.1002/aelm.202000880 . hal-03192473

\section{HAL Id: hal-03192473 \\ https://hal-univ-tours.archives-ouvertes.fr/hal-03192473}

Submitted on 29 Sep 2021

HAL is a multi-disciplinary open access archive for the deposit and dissemination of scientific research documents, whether they are published or not. The documents may come from teaching and research institutions in France or abroad, or from public or private research centers.
L'archive ouverte pluridisciplinaire HAL, est destinée au dépôt et à la diffusion de documents scientifiques de niveau recherche, publiés ou non, émanant des établissements d'enseignement et de recherche français ou étrangers, des laboratoires publics ou privés. 


\section{Fabrication of oriented n-type thermoelectric polymers by polarity switching in a DPP-based Donor-Acceptor copolymer doped with $\mathrm{FeCl}_{3}$}

Huiyan Zeng(1), Mohammed Mohammed (2), Viktoriia Untilova(1), Olivier Boyron (3), Nicolas Berton (2), Patrice Limelette (4) and Bruno Schmaltz (2), Martin Brinkmann(1)

(1) Université de Strasbourg, CNRS, ICS UPR 22, F-67000 Strasbourg, France

(2) PS2M, Université de Tours, Parc de Grandmont, F-37200, Tours, France

(3) Université de Lyon 1, CPE Lyon, CNRS UMR 5265, Laboratoire de Chimie Catalyse Polymères et Procédés (C2P2), Bat 308F, 43 bd du 11 Novembre 1918, 69616 Villeurbanne, France.

(4) GREMAN, UMR 7347 CNRS-INSA-Université de Tours, Parc de Grandmont, F-37200 TOURS, FRANCE

Corresp. Authors :

$\underline{\text { Martin.brinkmann@ics-cnrs.unistra.fr }}$

bruno.schmaltz@univ-tours.fr

patrice.limelette@univ-tours.fr 


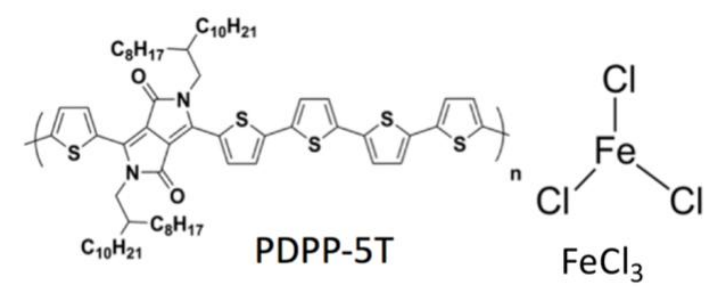

Polymer alignment

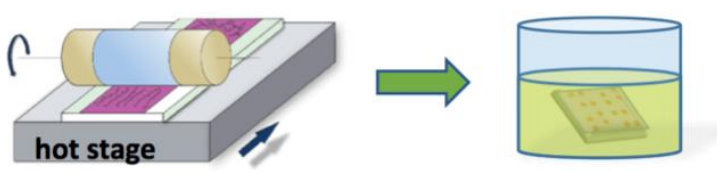

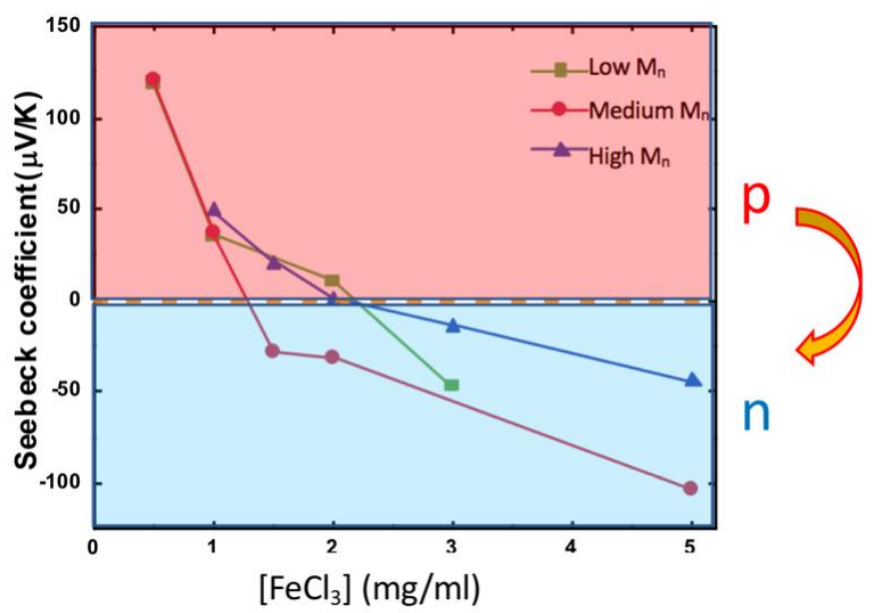

Abstract

$\mathrm{N}$-type doped polymers are key elements to fabricate all-polymer thermoelectric generators but they are challenging to produce. Herein a new strategy is proposed that is based on polarity switching upon doping of a donor-acceptor (D-A) copolymers based on diketopyrrolopyrrol (DPP) and quintethiophene (5T) with $\mathrm{FeCl}_{3}$. Polarity switching from p-type to n-type is observed upon increasing the doping concentration of $\mathrm{FeCl}_{3}$. We propose an analysis based on non-monotonic density of states which accounts for the main experimental trends and demonstrates that the polarity switch is governed by the electronic band filling that is determined by the dopant concentration. The influence of the curvature of the density of states is in addition discussed and a complete description of the doping induced transport regimes is proposed. This polarity switching depends on the molecular weight $\mathrm{M}_{n}$ of the polymer and shifts to higher $\mathrm{FeCl}_{3}$ concentrations with increasing $\mathrm{M}_{\mathrm{n}}$. This behavior is attributed to the change of the width of the density of states with $M_{n}$. The combination of polarity switching and alignment is a mean to produce n-type oriented and conducting polymers with enhanced power factors up to $10 \mu \mathrm{W} \cdot \mathrm{K}^{-2} \mathrm{~m}^{-1}$ along the chain direction. 


\section{Introduction.}

Doped polymer semiconductors (PSCs) have attracted increasing interest as potential thermoelectric (TE) materials that can be operated at temperatures below $200^{\circ} \mathrm{C}$ because of their mechanical flexibility, low cost and simple manufacturing as compared to inorganic semiconductors. ${ }^{[1,2]}$ Notably, the low thermal conductivity of conducting polymers is a priori favorable for attaining high TE performances which is assessed by the dimensionless figure of merit, $Z T=\frac{\alpha^{2} \sigma T}{\kappa}$, where $\alpha$, $\sigma, T$, and $\kappa$ are the Seebeck coefficient, electrical conductivity, absolute temperature, and the thermal conductivity, respectively.

In their seminal work, Crispin and coworkers demonstrated that precise control of the oxidation level of the poly(3,4-ethylenedioxythiophene) (PEDOT) chains allows to produce p-type polymers with remarkable thermoelectric efficiency $\mathrm{ZT}=0.25 .{ }^{[3]}$ Mastering the doping of PSCs is a central aspect in the development of new efficient thermoelectric polymers. At present, many p-type polymer materials have been identified with reasonable power factors up to $500 \mu \mathrm{W} \cdot \mathrm{K}^{-2} \cdot \mathrm{m}^{-1}$ using various material design strategies including e.g. polymer side chain engineering, polymer mixing, control of polymer crystallinity, optimization of the doping mechanism (solution and vapor phase) or polymer alignment. $(1,2,4-10)$ The situation for n-type polymers is less favorable as it is difficult to synthesize stable n-type polymers with low-enough LUMO level. Doping a classical n-type donor-acceptor copolymer such as poly[(N,N'-bis(2-octyldodecyl)-naphthalene-1,4,5,8bis(dicarboximide)-2,6-diyl] with $\quad$ 4-(1,3-dimethyl-2,3-dihydro-1H-benzoimidazol-2yl)phenyl)dimethylamine (n-DMBI) leads to limited charge conductivities of up to $510^{-3} \mathrm{~S} / \mathrm{cm}$ and power factors below $1 \mu \mathrm{W} \cdot \mathrm{K}^{-2} \cdot \mathrm{m}^{-1} \cdot{ }^{[11]}$ Pei and coworkers designed new n-type D-A copolymers

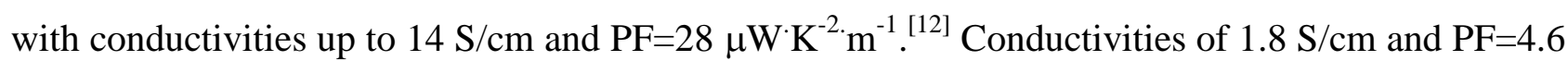
$\mu \mathrm{W} \cdot \mathrm{K}^{-2} \cdot \mathrm{m}^{-1}$ could be achieved by side chain engineering of P(NDIT2OD) using ethylene glycol side chains. ${ }^{[13]}$ Most interestingly the latter authors demonstrated that the n-type polymer can switch polarity upon increasing the doping concentration of n-DMBI. The Seebeck coefficient changed 
from negative to positive at a doping concentration of $35 \mathrm{~mol} \%$. It was explained by assuming hopping of holes between Charge Transfer Complex (CTC) states. Hwang et al. experienced a similar polarity switching form $\mathrm{n}$ to $\mathrm{p}$-type in poly(pyridiniumphenylene). The authors explained the polarity switching based on Ultraviolet Photoelectron Spectroscopy (UPS) experiments. ${ }^{[14]}$ They showed that when the LUMO is fully filled with electrons upon doping, it acts as a new HOMO with the LUMO+1.

Is it possible to use a similar polarity switching to produce n-type conducting polymer films starting from a p-type polymer upon doping? We have investigated this possibility for a DPP-based (D-A) copolymer doped with a strong Lewis acid, namely $\mathrm{FeCl}_{3}$ (see Figure 1). Diketopyrrolopyrrole (DPP) based copolymers are particularly attractive for organic field effect transistors (OFET) as well as organic photovoltaics (OPV) due to their high ambipolar charge carrier mobilities, broad absorption and high absorption coefficient. ${ }^{[15]}$ The DPP unit (electron acceptor $(\mathrm{A})$ ) has been combined with a large variety of co-monomers, such as phenyl, thiophene or thienothiophene ${ }^{[10]}$ working as (electron donor (D)). In this work, we focused on DPP-5T .

To further improve the potential TE properties of the films, we used alignment of the films by high temperature rubbing. As shown by Brinkmann and coworkers, alignment of polymers is an effective strategy to enhance TE properties as both conductivity and Seebeck coefficient can be enhanced simultaneously upon sequential doping of the oriented polymer semiconductors. In particular, for PBTTT, we demonstrated that record power factors beyond $1 \mathrm{~mW} \cdot \mathrm{K}^{-2} \mathrm{~m}^{-1}$ can be reached upon sequential doping of highly oriented $\mathrm{C}_{12}$-PBTTT with $\mathrm{FeCl}_{3}$.

In this study, we focus on PDPP-5T, which consists of alternating a DPP core unit and a quinquethiophene unit (5T) in the backbone (see Figure 1). The $\mathrm{C}_{20} \mathrm{H}_{41^{-}}$alkyl side chain are branched and located on the DPP units. The branching point in the side chain is on the second carbon atom. It helps to increase the polymers solubility and facilitate stronger intermolecular interaction between individual polymer chains with reduced pi -stacking distance. ${ }^{[14]}$ 


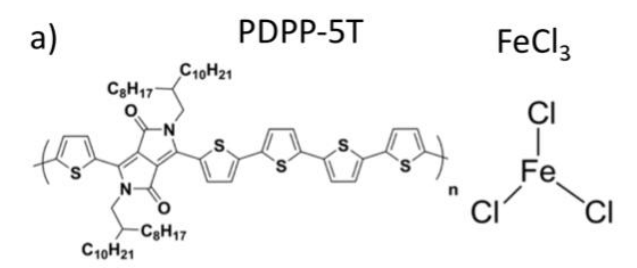

b)
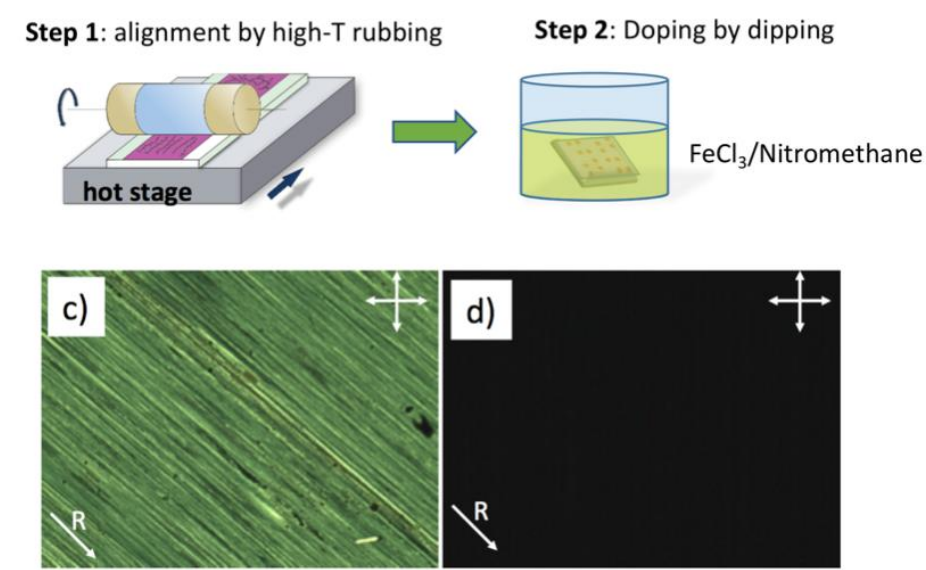

Figure 1. a) Chemical structure of Poly[[2,5-di(2-octyldodecyl)pyrrolo[3,4-c]-pyrrole-1,4(2H,5H)-

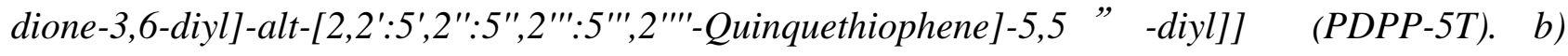
Method of film preparation by high-temperature rubbing to align PDPP-5T and sequential doping in a solution of $\mathrm{FeCl}_{3} /$ nitromethane. c) and d) POM images under crossed polarizers of the oriented thin films at $45^{\circ}$ and $0^{\circ}$ with respect to the rubbing direction $(R)$.

As a dopant, we used the Lewis acid $\mathrm{FeCl}_{3}$ that was previously used to dope PBTTT and other DPP-based systems such as PDPP-3T. ${ }^{[10,16,17]}$ Jung et al. have proved that PDPP-3T doped with $\mathrm{FeCl}_{3}(10 \mathrm{mM})$ can reach high conductivity values up to $55 \mathrm{~S} / \mathrm{cm}$ in non-oriented films for high molecular weight polymers. ${ }^{[16]}$ In this work, high temperature rubbing and sequentially doping were used to improve the TE properties of oriented films. ${ }^{[18,8]}$ High temperature rubbing was used to align the PDPP-5T along the rubbing direction, thus improving the charge carrier mobility that is dominated by charge transport along the polymer backbone. The structural and electronic modifications induced by doping oriented PDPP-5T films with $\mathrm{FeCl}_{3}$-doped were investigated by transmission electron microscopy, polarized UV-vis-NIR spectroscopy and conductivity and Seebeck coefficient measurements. 
As expected, alignment leads to improved TE properties since both conductivity and thermopower are enhanced along the rubbing direction. ${ }^{[5,16]}$ This contribution demonstrates that the TE properties depend strongly on $M_{n}$ with the best properties observed for medium- $M_{n}$ PDPP-5T. Moreover, we demonstrate that PDPP-5T shows a remarkable $\mathrm{p} \rightarrow \mathrm{n}$ polarity switching that depends on $\mathrm{M}_{\mathrm{n}}$. The polarity change is observed when the conductivity reaches a maximum as a function of $\mathrm{FeCl}_{3}$ concentration. For the p-type regime of transport, the oriented films show maximum conductivities $\sigma_{/ /}=327 \mathrm{~S} / \mathrm{cm}$ and $\mathrm{PF}=47 \mu \mathrm{W} \cdot \mathrm{K}^{-2} \cdot \mathrm{m}^{-1}$ whereas in the n-type regime, $\sigma / /=124 \mathrm{~S} / \mathrm{cm}$ and $\mathrm{PF}=10$ $\mu \mathrm{W} \cdot \mathrm{K}^{-2} \cdot \mathrm{m}^{-1}$.

\section{Results and discussion}

In the following, we will present subsequently, the spectroscopic signatures of doping, the structural evolution of the films upon doping and finally the thermoelectric properties of the doped films.

\section{1) UV-Vis-Near-Infrared (NIR) Spectroscopy}

Oriented films of PDPP-5T were prepared by rubbing at different temperatures between room temperature and $200^{\circ} \mathrm{C}$. Three $\mathrm{M}_{\mathrm{n}}$ fractions were obtained by Soxhlet extraction in acetone (low- $\mathrm{M}_{\mathrm{n}}$ ) chloroform (Medium- $\mathrm{M}_{\mathrm{n}}$ ) and ortho-dichlorobenzene $\left(\right.$ High $\left.-\mathrm{M}_{\mathrm{n}}\right)$. For each $\mathrm{M}_{\mathrm{n}}$, the best conditions of orientation in terms of rubbing temperature were determined by POM and polarized UV-vis-NIR spectroscopy. Indeed, it was demonstrated previously that the $\mathrm{M}_{\mathrm{n}}$-distribution determines the best

temperature for rubbing. ${ }^{[19]}$ The UV-Vis-NIR absorption spectra with two different polarization directions (parallel and perpendicular to the rubbing) give evidence for the orientation of the PDPP5T chains mainly along the rubbing direction (see Figure 2).

The spectra of PDPP-5T in thin films (low- $\mathrm{M}_{\mathrm{n}}$ sample) consist of the intramolecular charge transfer band with the $0-0$ and $0-1$ contributions at $778 \mathrm{~nm}$ and $700 \mathrm{~nm}$ and the $\pi-\pi^{*}$ absorption band at $457 \mathrm{~nm}$. It is also similar to that of PDPP-5T in solution in ODCB. The spectrum of the polymer changes slightly with $M_{n}$ in thin films. One can observe i) a 10nm blue shift of the 
intramolecular CT bands when $\mathrm{M}_{\mathrm{n}}$ increases and ii) a small shoulder around $\lambda=850 \mathrm{~nm}$ that is less absent for the low- $M_{n}$ sample. The presence of such a low energy band reminds the case of P(NDI2ODT2) whose polymorphism accounts for such spectroscopic features. Indeed, a segregated stacking of D and A units has been shown to result in the presence of a similar low energy component that was attributed to a segregated type of packing of D and A units. The presence of the $850 \mathrm{~nm}$ band might be an indication that DPP and 5T are stacked in segregated manner. ${ }^{[17,18,19]}$

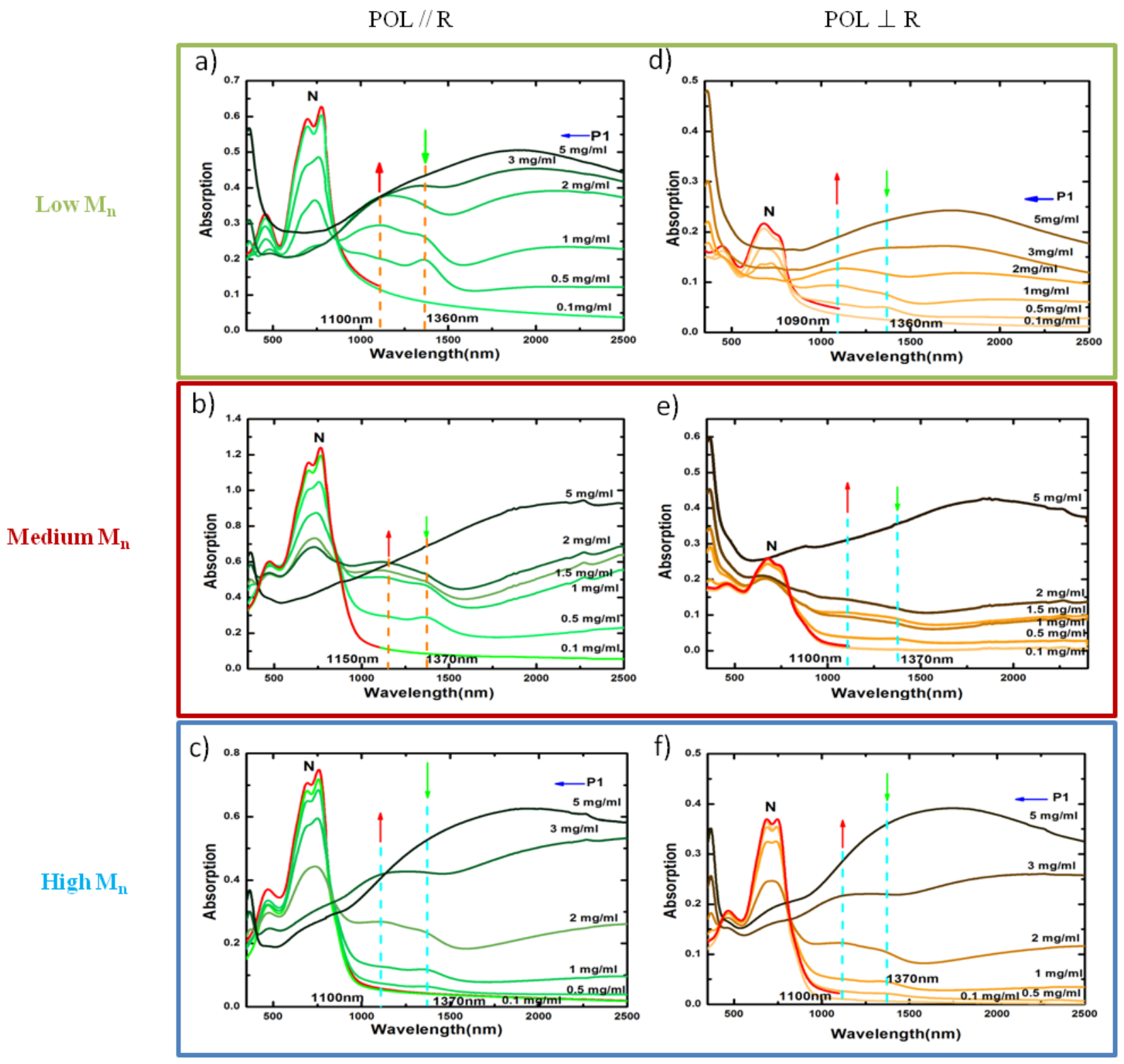

Figure 2. Evolution of the polarized UV-Vis-NIR spectra of oriented PDPP-5T films oriented by high-temperature rubbing and sequentially doped in solution of $\mathrm{FeCl}_{3} /$ nitromethane of increasing 
concentration for three samples with increasing molecular weight. The light polarization is parallel (//) to the rubbing direction $(a, b, c)$ or perpendicular to it $(\perp)(d, e, f)$.

As expected, the rubbing temperature leading to the best alignment depends strongly on $M_{n}$. However, contrary to previous findings for P3HT and PBTTT showing that orientation sets in at higher $T_{R}$ for higher $M_{n}$, it is observed that alignment is best for lower $T_{R}$ when $M_{n}$ increases. The best alignment is observed for the medium- $\mathrm{M}_{\mathrm{n}}$ sample with a maximum dichroic ratio $\mathrm{DR}=\mathrm{Abs} / / \mathrm{Abs}_{\perp}$ at $780 \mathrm{~nm}$ of 5.5 whereas both other samples show DR below 3 (see supporting information Figure S1).

Figure 2 shows the evolution of the UV-Vis-NIR spectra of the oriented PDPP-5T films upon doping with increasing $\mathrm{FeCl}_{3}$ solution in acetonitrile for the three $\mathrm{M}_{\mathrm{n}}$. The spectra in 2 , a, b and c correspond to the light polarization oriented parallel to the rubbing direction (POL//R) whereas in d,e and $\mathrm{f}$ the spectra for POL $\perp \mathrm{R}$ are shown. All samples are doped by dipping in the solutions for 10 s.

Let us first focus on the doping of the low- $\mathrm{M}_{\mathrm{n}}$ sample for POL//R. Three doping regimes can be distinguished. First, for $\left[\mathrm{FeCl}_{3}\right] \leq 1 \mathrm{mg} / \mathrm{ml}$, one observes the decrease in the neutral absorption of PDPP-5T (N band) that coincides with the appearance of three bands at $2200 \mathrm{~nm}, 1360 \mathrm{~nm}$ and $1100 \mathrm{~nm}$. The latter bands are dominant in the NIR domain. Similar features were observed upon doping a DPP-based polymer with $\mathrm{F}_{6} \mathrm{TCNNQ}$ and were assigned to a Charge Transfer complex (CTC) by Karpov et al. ${ }^{[20]}$ A similar assignment is possible in the present case. For $\left[\mathrm{FeCl}_{3}\right] \leq 3 \mathrm{mg} / \mathrm{ml}$, the UV-Vis-NIR spectra are dominated by two polaronic features P1 and P2 around 2000-220nm and 1100nm, similar to the bands seen upon doping polythiophenes such as P3HT and PBTTT. Interestingly, the increase of $\left[\mathrm{FeCl}_{3}\right]$ induces a progressive blue-shift of the P1 band. The position of polaronic features is usually related to the delocalization of the polarons. A blue shift is often associated to a localization of the polaron eventually due to enhanced Coulombic interactions between the polaron and the anion. ${ }^{[21,22]}$ Most interestingly, the CTC band at 1360nm 
tends to disappear or at least to merge with the $\mathrm{P} 2$ band with increasing $\left[\mathrm{FeCl}_{3}\right]$. It has also to be noticed that for $\left[\mathrm{FeCl}_{3}\right]=2 \mathrm{mg} / \mathrm{ml}$, the absorption of the neutral polymer band has almost fully disappeared, indicating that the polymer backbone is fully oxidized. Finally, for $\left[\mathrm{FeCl}_{3}\right] \geq 5 \mathrm{mg} / \mathrm{ml}$, the absorption is dominated by a broad absorption band centered at $1900 \mathrm{~nm}$. As a general observation, the vibronic structure of the neutral polymer band is substantially modified during the doping process. For $\left[\mathrm{FeCl}_{3}\right]=0.5 \mathrm{mg} / \mathrm{ml}$, the $0-1$ absorption band of PDPP-5T is seen as a shoulder beside the $0-0$ band. This loss of sharp vibronic structure suggests some inhomogeneous broadening possibly related to some disordering in the stacks of backbones upon doping.

For POL $\perp \mathrm{R}$, the same polaronic and CTC features seen for POL//R are observed but with a much smaller intensity, indicating that they are both polarized in the direction of the polymer backbone.

The comparison of the dependence of the spectral features as a function of $\left[\mathrm{FeCl}_{3}\right]$ for different $M_{n}$ samples is also instructive. The CTC and polaronic features seen for the low- $M_{n}$ sample are also seen for the higher- $\mathrm{M}_{\mathrm{n}}$ samples. For all samples, the increase in $\left[\mathrm{FeCl}_{3}\right]$ results in a blue-shift of the P1 band. There are however certain differences. The intensity of the CTC band at $1360 \mathrm{~nm}$ tends to decrease when $\mathrm{M}_{\mathrm{n}}$ increases (this is particularly visible for $\left[\mathrm{FeCl}_{3}\right]=0.5 \mathrm{mg} / \mathrm{ml}$ ). The $\mathrm{P} 1$ band is more red-shifted for the medium and high- $\mathrm{M}_{\mathrm{n}}$ samples for $\left[\mathrm{FeCl}_{3}\right]=1-2 \mathrm{mg} / \mathrm{ml}$ than for the low-M sample.

\section{2) Structural variation with doping concentration.}

The impact of doping on the structure of the oriented PDPP-5T films was followed by TEM. Regarding the undoped polymer films, they show similar ED patterns for all $\mathrm{M}_{\mathrm{n}}$. Electron diffraction (ED) indicates that PDPP5T adopts a classical layered structure made of $\pi$-stacked PDPP5T backbones separated by layers of alkyl side chains. The inter-layer spacing along alkyl side chains $d_{100}$ and the $\pi$-stacking periodicity $d_{020}$ vary slightly with $M_{n}$. As a trend, $d_{100}$ increases 
and $\mathrm{d}_{020}$ decreases with increasing $\mathrm{M}_{\mathrm{n}}\left(\mathrm{d}_{100}=2.12 \mathrm{~nm}\right.$ and $\mathrm{d}_{020}=0.375 \mathrm{~nm}$ for $\mathrm{M}_{\mathrm{n}}=11.2 \mathrm{kDa}$ and $\mathrm{d}_{100}=1.84 \mathrm{~nm}$ and $\mathrm{d}_{020}=0.343 \mathrm{~nm}$ for $\left.\mathrm{M}_{\mathrm{n}}=7.35 \mathrm{kDa}\right)$.

The ED patterns of rubbed PDPP5T films (see Figure 3) shows two equatorial $d_{100}$ and $d_{020}$ reflections. The presence of both peaks indicates coexistence of face-on and edge-on domains sharing the same in-plane orientation of the chains parallel to the rubbing direction $\mathrm{R}$. The proportion of edge-on versus face-on crystals depends on $M_{n}$ and the temperature $T_{R}$ used to achieve maximal orientation. As observed in most polymer semiconductors aligned by high- $\mathrm{T}_{\mathrm{R}}$, high $T_{R}$ favors edge-on crystals. ${ }^{18,23}$ Since the low- $M_{n}$ films were rubbed at $200^{\circ} \mathrm{C}$, medium- $\mathrm{M}_{n}$ films at $120^{\circ} \mathrm{C}$ and high- $\mathrm{M}_{\mathrm{n}}$ samples at $60^{\circ} \mathrm{C}$, the proportion of edge-on crystals is highest for the low- $\mathrm{M}_{\mathrm{n}}$ sample. The absence of any reflection along the chain direction indicates no periodic order in this direction. This means that the 5T and DPP blocks tend to $\pi$-stack in a statistic manner without segregation between the two blocks. ${ }^{[19]}$ The branched nature of side chains on the DPP might be responsible for this lack of ordering along the backbone direction. It is worth mentioning that the presence of statistical disorder in the stacking along the chain direction does not impede the chains to $\pi$-stack.

Qualitatively, the evolution of the ED pattern upon increasing $\mathrm{FeCl}_{3}$ concentration shows following trends (see Figure 3a-c): i) the intensity of the 100 is enhanced for $\left[\mathrm{FeCl}_{3}\right]=1 \mathrm{mg} / \mathrm{ml}$ with respect to the pristine samples, ii) it decreases for larger doping concentrations. It is likely that doping at low concentration helps induce some order in the polymer, a situation encountered for the smectic-like phase of P3HT doped with $\mathrm{F}_{4}$ TCNQ and also for regio-random P3HT. ${ }^{[23]}$ In contrast, the lamellar order seems to be perturbed by the introduction of higher dopant concentrations. Doping also induces changes of the lattice parameters of the PDPP5T crystals. For the low-M sample, $\mathrm{d}_{100}$ increases from $1.84 \pm 0.03 \mathrm{~nm}$ in pristine samples and saturates to $2.04 \pm 0.03 \mathrm{~nm}$ for $5 \mathrm{mg} / \mathrm{ml} \mathrm{doped}$ films whereas for the high- $\mathrm{M}_{\mathrm{n}}$ sample the increase is from $2.15 \pm 0.05 \mathrm{~nm}$ to $2.36 \pm 0.05 \AA$ (see Figure 3.d and 3.e). In addition, doping induces also a contraction of the lattice along the $\pi$-stacking. The 
$\pi$-stacking distance becomes particularly small in the Low- $\mathrm{M}_{\mathrm{n}}$ sample doped with $5 \mathrm{mg} / \mathrm{ml} \mathrm{FeCl} \mathrm{F}_{3}$ and reaches $0.334 \pm 0.005 \mathrm{~nm}$. Similarly to other polymer/dopant systems such as $\mathrm{P} 3 \mathrm{HT} / \mathrm{F}_{4} \mathrm{TCNQ}$ and $\mathrm{FeCl}_{3} / \mathrm{PBTTT}$, this structural change can be interpreted by the intercalation of dopant molecules in the polymer lattice. ${ }^{[8-10]}$ In the case of $\mathrm{FeCl}_{3}$ doping, it was suggested that both $\mathrm{FeCl}_{2}$ and $\mathrm{FeCl}_{4}{ }^{-}$ species are incorporated into the alkyl side chain layers upon doping of polymer films (see Figure 3.f). ${ }^{[10]}$
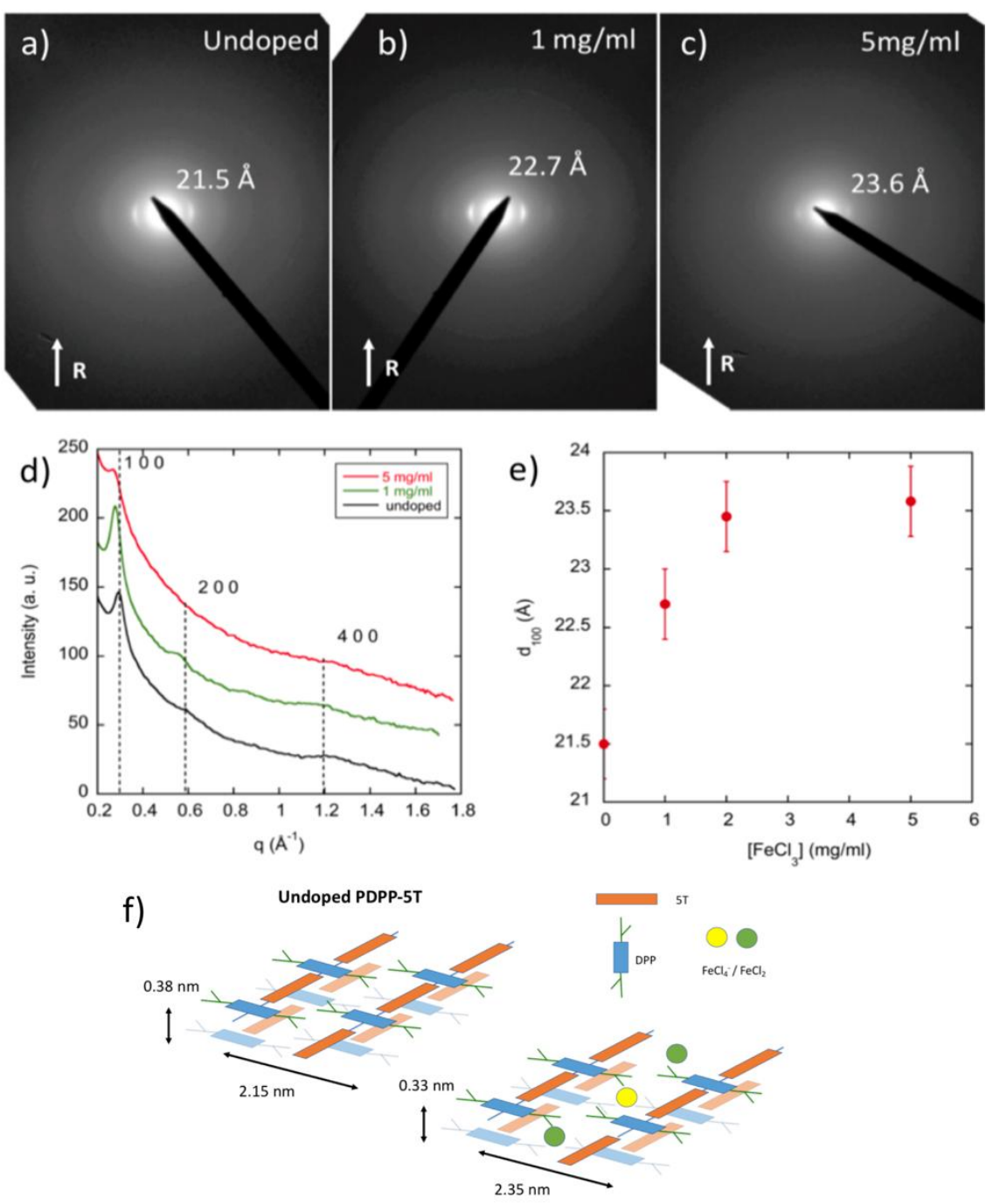

$\mathrm{FeCl}_{3}$-doped PDPP-5T

Figure 3. Evolution of the electron diffraction pattern of oriented PDPP-5T (High $\left.M_{n}\right)\left(T_{R}=125^{\circ} \mathrm{C}\right)$ as a function of doping concentration with $\mathrm{FeCl}_{3} . \mathrm{R}$ is the rubbing direction. a) as-rubbed films, $b$ ) doped with $1 \mathrm{mg} / \mathrm{ml}, \mathrm{c}$ ) doped with $5 \mathrm{mg} / \mathrm{ml}$. d) Equatorial section profiles of the ED patterns. e) variation of $d_{100}$ with increasing doping concentration. f) Schematic illustration of the $\mathrm{FeCl}_{3}$ intercalation in the side chain layers of PDPP5T. 


\section{3) Charge transport and Thermopower measurements.}

\section{a) Non-oriented films.}

Before analyzing the TE properties of aligned films, we tested the non-oriented PDPP-5T films as a function of $M_{n}$. The conductivity shows for all $M_{n}$ fractions the same trend i.e. an increase until a maximum conductivity followed by a decrease for high $\left[\mathrm{FeCl}_{3}\right]$. The low- $\mathrm{M}_{\mathrm{n}}$ fraction shows a maximum conductivity of up to $47 \mathrm{~S} / \mathrm{cm}$ whereas the highest conductivity of $209 \mathrm{~S} / \mathrm{cm}$ is obtained for the medium- $M_{n}$ fraction. As seen in Figure $4 a$, the main difference between samples is the concentration of $\mathrm{FeCl}_{3}$ for which the maximum conductivity is observed. As a general rule, the higher $\mathrm{M}_{\mathrm{n}}$, the larger the value of $\left[\mathrm{FeCl}_{3}\right]$ corresponding to the maximum of conductivity: typically $\sigma$ reaches a maximum for $\left[\mathrm{FeCl}_{3}\right]=1 \mathrm{mg} / \mathrm{ml}$ for low- $\mathrm{M}_{\mathrm{n}}$ PDPP-5T versus $5 \mathrm{mg} / \mathrm{ml}$ for the high- $\mathrm{M}_{\mathrm{n}}$ sample.

More interesting is the evolution of the Seebeck coefficient versus $\left[\mathrm{FeCl}_{3}\right]$. As seen in Figure $4 \mathrm{~b}$, the Seebeck coefficient tends to decrease with increasing $\left[\mathrm{FeCl}_{3}\right]$ as observed for most semiconductors upon doping. However, all three samples exhibit a characteristic polarity switch from positive to negative indicating a change of sign of the majority charge carriers. In other words, upon doping PDPP-5T with $\mathrm{FeCl}_{3}$, the originally p-type polymer becomes n-type at a given doping level. Interestingly, the value of $\left[\mathrm{FeCl}_{3}\right]$ corresponding to the polarity switch depends on the $\mathrm{M}_{\mathrm{n}}$ of the polymer: the higher $\mathrm{M}_{\mathrm{n}}$, the higher the value of $\left[\mathrm{FeCl}_{3}\right]$ corresponding to the polarity change. 
a)

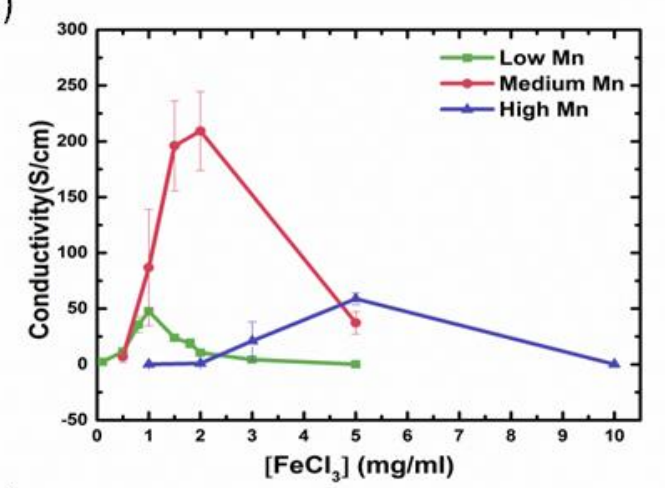

b)

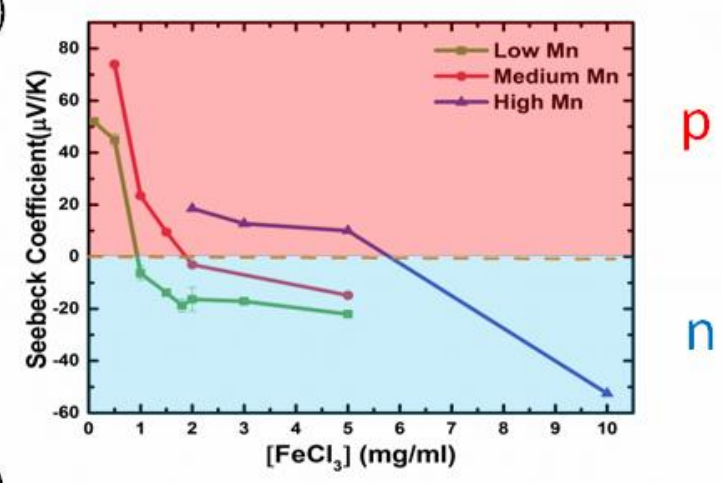

c)

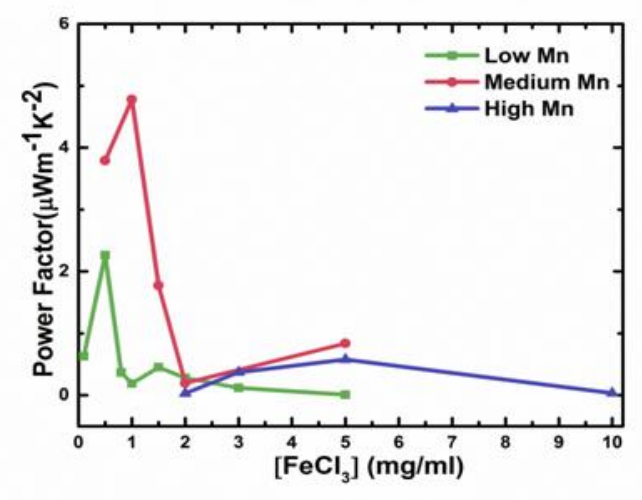

Figure 4. Evolution of the conductivity (a), of the Seebeck coefficient (b) and the power factor (PF)

(c) in non-oriented PDPP-5T thin films as a function of $\mathrm{FeCl}_{3}$ concentration for three different molecular weights. Note the polarity switch in the Seebeck coefficient with increasing $\left[\mathrm{FeCl}_{3}\right]$.

\section{b) Oriented PDPP-5T films.}

Let us focus on the oriented PDPP-5T films. As seen in Figure 5, the trends observed for the nonoriented samples are also valid for the oriented films. Regarding the charge conductivity along the chain direction $\sigma_{/ /}$, the medium- $\mathrm{M}_{\mathrm{n}}$ sample is able to reach conductivities of the order of $327 \pm 80$ $\mathrm{S} / \mathrm{cm}$ rather close to those of the high $-\mathrm{M}_{\mathrm{n}}$ sample (close to $177 \pm 14 \mathrm{~S} / \mathrm{cm}$ ) along the chain direction. The conductivity in the low $-\mathrm{M}_{\mathrm{n}}$ samples is substantially lower and of the order of $88 \pm 16 \mathrm{~S} / \mathrm{cm}$. 
Clearly, $\sigma_{/ /}$for the medium and High- $\mathrm{M}_{\mathrm{n}}$ samples are improved by alignment over non-oriented films.

$\mathrm{POL} / / \mathrm{R}$

a)

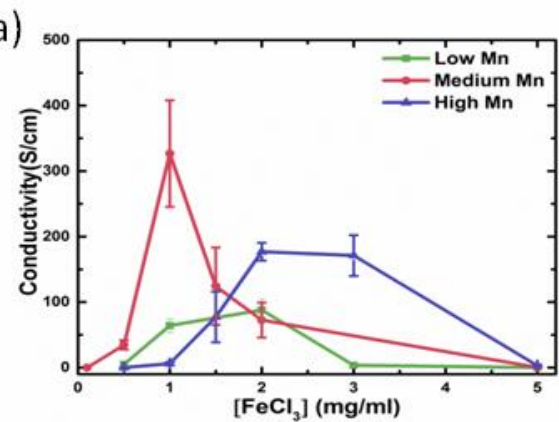

b)
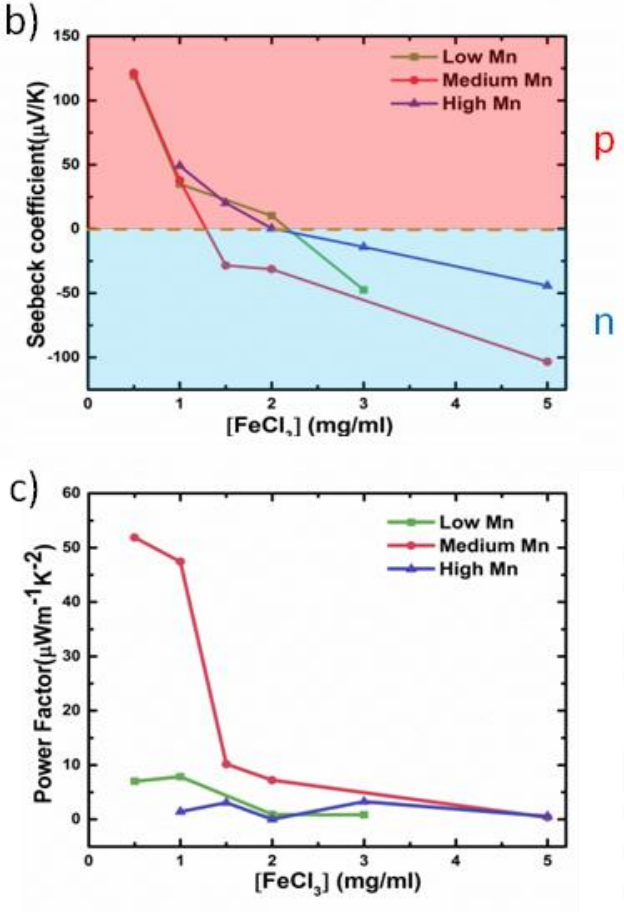

$\mathrm{POL} \perp \mathrm{R}$

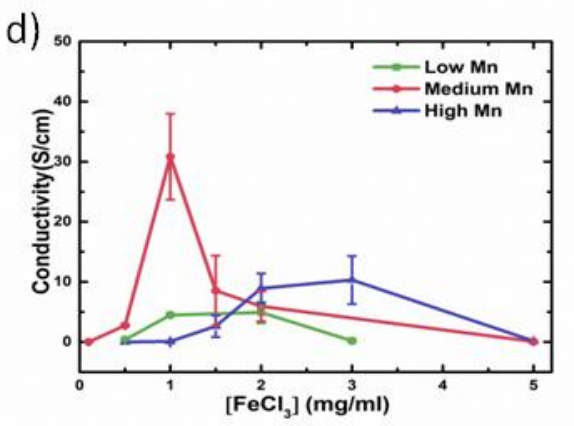

e)
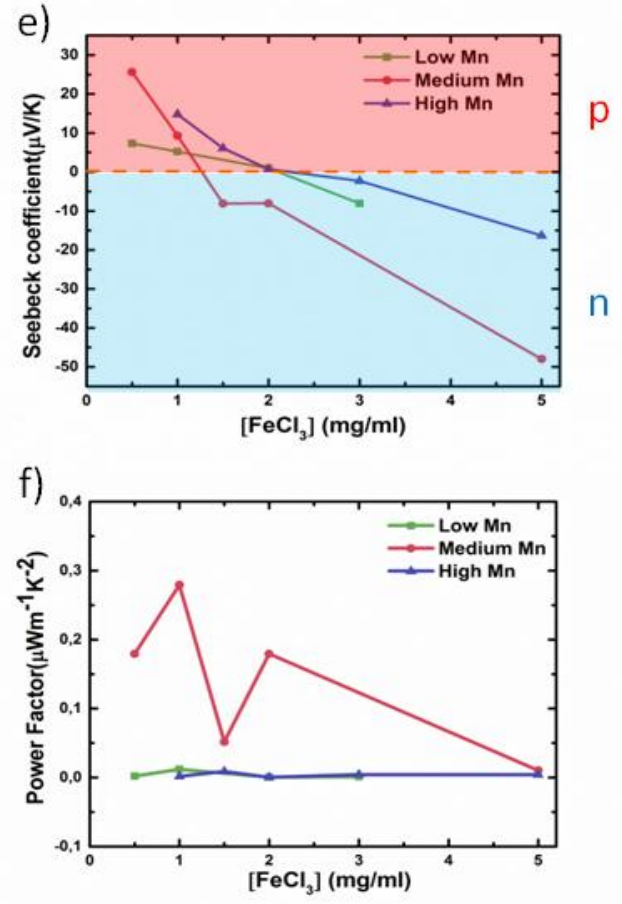

Figure 5. Evolution of the charge conductivity ( $a$ and $d$ ), of the thermopower $(b$ and $e$ ) and the power factor (c and f), as a function of $\mathrm{FeCl}_{3}$ concentration measured in the direction parallel $(a, b, c)$ and perpendicular $(d, e, f)$ to the chains for oriented thin films of PDPP-5T of different molecular weights.

The reason why the conductivity of low- $\mathrm{M}_{\mathrm{n}}$ lies clearly below the other two samples may be related to the interconnectivity between ordered domains. Indeed, in P3HT OFETs it has been shown that charge transport is determined by the presence of tie-chains that interconnect the crystalline domains. Low- $\mathrm{M}_{\mathrm{n}}$ P3HT (5-7kDa) show very low charge mobilities versus medium and high- $\mathrm{M}_{\mathrm{n}}$ 
P3HT because tie-chains are essentially absent in low- $\mathrm{M}_{n}$ polymers. ${ }^{[24]} \mathrm{A}$ similar reasoning is possible in the present case for doped PDPP5T because all samples show similar levels of charge carrier densities (thickness-normalized polaron absorbances are similar for all $\mathbf{M}_{n}$ samples). Accordingly, the one-order of magnitude difference in conductivity must be related to differences in carrier mobilities between the different $\mathrm{M}_{\mathrm{n}}$ samples. Regarding the conductivity perpendicular to the chains $\sigma_{\perp}$, the same trend is observed as for $\sigma_{/ /}$as a function of $M_{n}$ : the medium- $M_{n}$ sample shows the largest conductivity that is similar to that of the non-oriented sample.

For the Seebeck coefficient of the aligned films (see Figure $5 \mathrm{~b}$ and d), the polarity switch is observed for all $\mathrm{M}_{\mathrm{n}}$ samples as for the non-oriented films. The polarity switch is observed in both directions parallel and perpendicular to the rubbing direction $\mathrm{R}$. The values of $\left[\mathrm{FeCl}_{3}\right]$ corresponding to the polarity change are slightly different from the non-oriented films. This might be due to differences in film morphology and structure e.g. the proportion of face-on domains that is more important in rubbed films and that may ease the dopant diffusion in the thin films.

\section{Origin of the polarity switching.}

\section{a) Impact of the analytical form of the dos.}

Whereas the correlation between the electrical conductivity and the thermopower has been widely addressed in regimes where the latter keeps the same $\operatorname{sign}{ }^{[25]}$, we investigate hereafter the specific case of the doping induced polarity switch that is characterized by the sign change of the thermopower associated to a maximum of conductivity. We propose an analysis based on nonmonotonic density of states (dos) which accounts for the main experimental trends and demonstrates that the polarity switch is governed by the electronic filling, namely that it is doping induced.

The transport phenomena in solids are generally successfully described by the linearized semi-classical Boltzmann equation in the frame of the relaxation time approximation. By taking into 
account both thermal and chemical potential gradients, the transport coefficients such as the electrical conductivity $\sigma$ and the thermopower $\alpha$ can be written as a function of the kinetic coefficients $L_{i j, x}$ in the x direction, which involve the following transport integrals: ${ }^{[26,27]}$

$$
\begin{gathered}
L_{i j, x}=T \int \tau_{E} v_{x, E}^{2}(E-\mu)^{i+j-2}\left(\frac{-\partial f_{E}}{\partial E}\right) g_{E} d E \\
\sigma=\frac{q^{2} L_{11}}{T} \\
\alpha=\frac{L_{12}}{q T L_{11}}
\end{gathered}
$$

with the energy dependent relaxation time $\tau_{\mathrm{E}}$, the velocity $v_{x, E}$, the density of states $\mathrm{g}_{\mathrm{E}}$ and the Fermi-Dirac statistic $\mathrm{f}_{\mathrm{E}}$, $\mathrm{T}$ being the temperature, $\mathrm{q}$ the electron charge and $\mu$ the chemical potential.

From a general point of view, all the energy dependences need to be taken into account according to the specific scattering mechanisms even if in a degenerate system the standard Sommerfeld's expansion allows to develop the transport integrals around the Fermi energy $\mathrm{E}_{\mathrm{F}}$. Nevertheless, in order to focus on the doping induced sign change of the thermopower correlated to the maximum of the conductivity, one may solely retain the energy dependence in the density of states (dos) by neglecting the energy-dependences of both the electronic relaxation time $\tau_{\mathrm{F}}$ and velocity $v_{F}$ at the Fermi level. This assumes that in such a regime the doping induced variation of the dos as a function of the energy dominates over the other ones. Therefore, the Sommerfeld expansion allows to relate explicitly the charge conductivity $\sigma$ and the thermopower $\alpha$ to the dos following:

$$
\begin{aligned}
& \sigma=\frac{q^{2} \tau_{F} v_{F}^{2}}{3} g_{E_{F}} \\
& \alpha=\frac{\pi^{2}}{3} \frac{k_{B}}{q} k_{B} T\left(\frac{\partial \ln \left(g_{E}\right)}{\partial E}\right)_{E_{F}}
\end{aligned}
$$

One recovers here an expression of the thermopower equivalent to the Mott formula ${ }^{[28]}$ with constant $\tau_{\mathrm{F}}$ and $v_{F}$. Interestingly, these simple relations allow now to investigate the influence of non-monotonic energy dependence in the dos on the transport coefficients. In order to investigate 
the effects of finite bandwidth and curvature, three kinds of dos can be considered including a Gaussian, $\mathrm{g}_{\mathrm{G}}$, a semi-circular, $\mathrm{g}_{\mathrm{sc}}$, and a modified parabolic dos $\mathrm{g}_{\mathrm{p} \pm}$ as written below.

$$
\begin{gathered}
g_{G}(E)=g_{G, W} e^{-\left(E / W_{ \pm}\right)^{2}} \\
g_{s c}(E)=g_{s c, W} \sqrt{1-\left(E / W_{ \pm}\right)^{2}} \\
g_{p \pm}(E)=g_{p, W} \sqrt{1-\left(1-\left(1 \mp E / W_{ \pm}\right)^{4}\right)^{2}}
\end{gathered}
$$

For the semi-circular and modified parabolic dos, W corresponds to the half-bandwidth strictly speaking whereas for the Gaussian distribution, the width $\left(W=\sqrt{2} \sigma_{d o s}\right)$ is usually ascribed to an energetic disorder. ${ }^{[29-32]}$ In the following analysis, $g_{G, W}, g_{s c, W}$ and $g_{p, W}$ are normalization constants, and the subscripts of $\mathrm{W}(-$ and + ) are used to take into account a possible electron-hole asymmetry according to the energy sign.

Figure 6.a displays the energy dependence of these density of states, in reduced unit, including the Gaussian, the semi-circular and the modified parabolic dos. These dos all exhibit a maximum, here defined at zero energy to facilitate their comparison, which separates the electron side $(E<0)$ from the hole one $(E>0)$. 

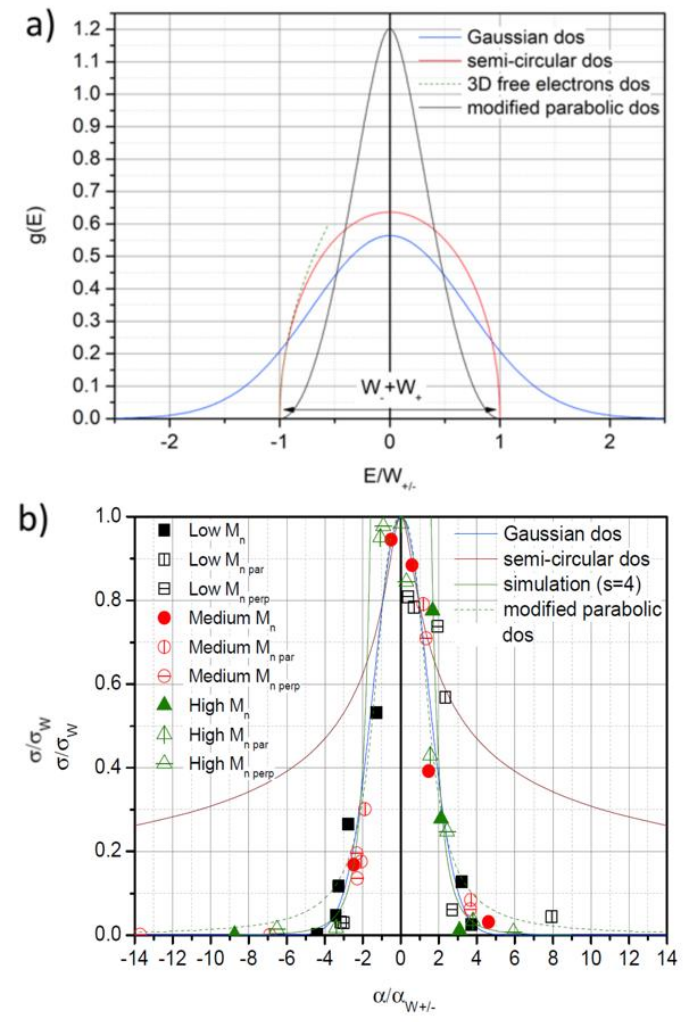

Figure 6. a) Energy-dependence in reduced units of the Gaussian, the semi-circular and the modified parabolic density of states (dos). Note that the semi-circular dos varies as the $3 D$ free electron's one near its band edges. b) Experimental normalized conductivity of $\mathrm{FeCl}_{3}$-doped PDPP$5 T$ films as a function of the normalized thermopower compared with theoretical predictions associated to Gaussian, semi-circular or modified parabolic dos. The behavior expected according to the scaling relationship $\alpha \propto \sigma^{-1 / s}$ with $s=4$ is also shown with adjusted normalization constants. The filled symbols correspond to non-oriented compounds and the open symbols to the oriented films.

Whereas the energy dependence of the Gaussian dos is unrestricted, making the latter appropriate to the description of broad electronic energy distributions, the other two dos are limited by their bandwidth (see Figure 6.a). Interestingly, the semi-circular dos varies near its band edges as $\sqrt{1 \mp E / W_{ \pm}}$namely as the dos of three-dimensional free carriers (electrons or holes depending on the energy sign). This makes it a natural extension of the free electron model involving a finite 
bandwidth. In strong contrast, the modified parabolic dos varies quadratically near its band edges, namely as $\left(1 \mp E / W_{ \pm}\right)^{2}$ and it is built by analogy with the semi-circular dos in order to display a maximum at zero energy. Due to its quadratic dependence near the edges, the modified parabolic dos has the same kind of curvature than the Gaussian dos in contrast to the semi-circular one. Nevertheless, it is defined, as previously emphasized, over a finite bandwidth as the semi-circular dos and in contrast to the Gaussian one. Additionally, it has been shown recently that a parabolic dos could be one of the microscopic ingredients allowing to justify the unusual scaling relationship between the thermopower and the electrical conductivity such as $\alpha \propto \sigma^{-1 / s}$ with $\mathrm{s}=4$. ${ }^{[25,33]}$

Analytical expressions of conductivity and thermopower can then be given as a function of the Fermi energy in each case by introducing the dimensional quantities $\sigma_{\mathrm{W}}$ and $\alpha_{\mathrm{W} \pm}$ which are defined positive. Table 1 collects the equations of the charge conductivity and Seebeck coefficients for the three type of dos versus Fermi energy $E_{\mathrm{F}}$.

Table 1. Correspondance between the analytical forms of the density of states (dos) and the conductivity and Seebeck coefficient as a function of Fermi energy $\mathrm{E}_{\mathrm{F}}$.

\begin{tabular}{|c|c|c|}
\hline Dos & Carrier conductivity & Seebeck coefficient \\
\hline Gaussian & $\sigma_{G}=\sigma_{G, W} e^{-\left(E_{F} / W_{ \pm}\right)^{2}}$ & $\alpha_{G}=\alpha_{W \pm}\left(2 E_{F} / W_{ \pm}\right)$ \\
\hline Semi-circular & $\sigma_{s c}=\sigma_{s c, W} \sqrt{1-\left(E_{F} / W_{ \pm}\right)^{2}}$ & $\alpha_{s c}=\alpha_{W \pm} \frac{\left(E_{F} / W_{ \pm}\right)}{1-\left(E_{F} / W_{ \pm}\right)^{2}}$ \\
\hline ModifiedParabolic & $\sigma_{p \pm}(E)=\sigma_{p, W} \sqrt{1-\left(1-\left(1 \mp E_{F} / W_{ \pm}\right)^{4}\right)^{2}}$ & $\alpha_{p \pm}=\alpha_{W \pm} \frac{ \pm 4\left(1 \mp E_{F} / W_{ \pm}\right)^{3}\left(1-\left(1 \mp E_{F} / W_{ \pm}\right)^{4}\right)}{\left(1-\left(1-\left(1 \mp E_{F} / W_{ \pm}\right)^{4}\right)^{2}\right)}$ \\
& & \\
\hline
\end{tabular}

Due to the choice of the energy origin, it is already clear that at half filling in the symmetric case, the Fermi energy is zero and the maximum of conductivity $\sigma_{\mathrm{W}}$ coincides with the cancellation of the thermopower. 
Besides, the latter relations allow to write the conductivity as a function of the thermopower in case of a Gaussian or semi-circular dos.

$$
\begin{gathered}
\sigma_{G}=\sigma_{G, W} e^{-\left(\alpha_{G} / 2 \alpha_{W \pm}\right)^{2}} \\
\sigma_{s c}=\sigma_{s c, W} \sqrt{1-1 / 4\left(\alpha_{W \pm} / \alpha_{s c}\right)^{2}\left(1-\sqrt{1+4\left(\alpha_{s c} / \alpha_{W \pm}\right)^{2}}\right)^{2}}
\end{gathered}
$$

These expressions indicate that the divergence of the thermopower is associated to the vanishing of the conductivity at the band edges when $\left|\mathrm{E}_{\mathrm{F}} / \mathrm{W}_{ \pm}\right|=1$, whatever the thermopower sign. So, in order to address the origin of the thermopower sign change and to test the relevance of such models, the experimental data were plotted in Fig. 6.b using the normalized conductivity and thermopower for all the investigated PDPP5T polymers. Please note that a single set of normalization constants has been used as free parameters for each polymer sample. When necessary, two different thermopower

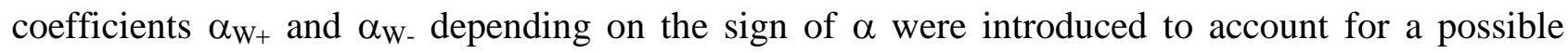
electron-hole asymmetry. The obtained values are collected in table 2.

It is worth mentioning that figure 6.b displays the normalized conductivity as a function of thermopower in order to focus on the polarity switch regime. First, it appears that the investigated non-monotonic dos account successfully for the thermopower sign change correlated to the maximum of conductivity. However, the range of agreement depends on the dos. For instance, the agreement between the semi-circular dos prediction and the experimental data is limited to a restricted range of conductivities such as $\sigma / \sigma_{\mathrm{w}}>0.7$. In strong contrast, the modified parabolic dos provides a much better agreement down to $\sigma / \sigma_{\mathrm{W}}>0.15$. It is however the Gaussian dos that yields the best agreement and reproduces nicely the polarity switch regime. This observation suggests that the Gaussian dos is best adapted to describe the electronic properties in conducting polymers as discussed in the literature. ${ }^{[23-26]}$ 
Some general trends and information can be gained from the fitting parameters used to account for the $\sigma-\alpha$ correlations for the different doped PDPP-5T films. Firstly, the extracted values of the normalization coefficients $\sigma_{\mathrm{W}}$ indicate the maximum value of conductivity according to the maximum of the dos. For all oriented PDPP-5T films, the values of $\sigma_{\mathrm{W}}$ in table 2 are larger (smaller) in the direction parallel (perpendicular) to the rubbing with respect to the values for non-oriented films. Moreover, the coefficient $\alpha_{W \pm}$ used to normalize the thermopower can be used to extract the width of the Gaussian distribution $\sigma_{\mathrm{dos}}{ }^{+}$. As seen from Table 2, the width $\sigma_{\mathrm{dos}}{ }^{+}$is systematically lower (larger) in the direction parallel (perpendicular) as compared to non-oriented films. Since this width is usually ascribed to an energetic disorder, this appears as a direct consequence of the orientation process with lower disorder in the parallel direction compared with the perpendicular direction. While a unique coefficient $\alpha_{\mathrm{W}}$ is mainly enough to normalize the data, one can refine the normalization by distinguishing the electron-like and the hole-like sides of the thermopower with possibly different coefficients $\alpha_{W \pm}$. As displayed in the table 1, such a procedure suggests a larger distribution width for the holes in the non-oriented Low $M_{n}$ compound and in the Medium $M_{n}$ compounds implying then an electron-hole asymmetry and lower energetic disorder.

Table 2. Summary of the parameters used to normalize the experimental data in the Fig. 7 for nonoriented and aligned PDPP-5T films ( $\mathrm{R}$ is the rubbing direction). The width of the Gaussian distribution $\sigma_{\text {dos }}$ is inferred from the normalization coefficient $\alpha_{W \pm}=\frac{\pi^{2}}{3} \frac{k_{B}}{|q|} \frac{k_{B} T}{W_{ \pm}}$with $W_{ \pm}=\sqrt{2} \sigma_{d o s} \pm$ where the minus sign (respectively plus) stands for the negative (positive) values of the electronlike (hole-like) thermopower. Note that an electron-hole asymmetry is here found in the nonoriented Low $\mathrm{M}_{\mathrm{n}}$ compound and in the Medium $\mathrm{M}_{\mathrm{n}}$ compounds (in italic).

\begin{tabular}{|c|c|c|c|c|c|c|c|c|c|}
\hline Sample & \multicolumn{3}{|c|}{ Low $\mathrm{M}_{\mathrm{n}}$} & \multicolumn{3}{c|}{ Medium $\mathrm{M}_{\mathrm{n}}$} & \multicolumn{3}{c|}{ High $\mathrm{M}_{\mathrm{n}}$} \\
\cline { 2 - 7 } & $\begin{array}{c}\text { Non- } \\
\text { oriented }\end{array}$ & $/ / \mathrm{R}$ & $\perp \mathrm{R}$ & $\begin{array}{c}\text { Non- } \\
\text { oriented }\end{array}$ & $/ / \mathrm{R}$ & $\perp \mathrm{R}$ & $\begin{array}{c}\text { Non- } \\
\text { oriented }\end{array}$ & $/ / \mathrm{R}$ & $\perp \mathrm{R}$ \\
\hline
\end{tabular}




\begin{tabular}{|c|c|c|c|c|c|c|c|c|c|}
$\begin{array}{c}\sigma_{\mathrm{W}} \\
(\mathrm{S} / \mathrm{cm})\end{array}$ & 89.5 & 112 & 6.1 & 221 & 413 & 43.5 & 75 & 180 & 10.5 \\
\hline $\begin{array}{c}\alpha_{\mathrm{W}-} \\
(\mu \mathrm{V} / \mathrm{K})\end{array}$ & 5 & 15 & 2.7 & 6 & 15 & 3.5 & 6 & 13 & 2.5 \\
\hline $\begin{array}{c}\sigma_{\text {dos- }} \\
(\mathrm{eV})\end{array}$ & 1.04 & 0.35 & 1.92 & 0.87 & 0.35 & 1.48 & 0.87 & 0.4 & 2.08 \\
\hline $\begin{array}{c}\alpha_{\mathrm{W}+} \\
(\mu \mathrm{V} / \mathrm{K})\end{array}$ & 14 & 15 & 2.7 & 16 & 33 & 7 & 6 & 13 & 2.5 \\
\hline $\begin{array}{c}\sigma_{\text {dos+ }} \\
(\mathrm{eV})\end{array}$ & 0.37 & 0.35 & 1.9 & 0.32 & 0.16 & 0.74 & 0.87 & 0.4 & 2.1 \\
\hline
\end{tabular}

As previously emphasized, the Gaussian dos provides thus a satisfactory description of the correlation between the electrical conductivity and the thermopower including consistent microscopic characteristics. It is instructive to compare the values of the width of the Gaussian dos with the values found in the literature for similar DPP polymers. In pristine films of DPP-DTT, the width of the dos describing energetic disorder was extracted by using different methods. ${ }^{[34-36]}$ The efficiency of photogenerated charge separation yielded $\sigma_{\text {dos }+}=0.18 \pm 0.02 \mathrm{eV} .{ }^{[34]}$ Lower values $\sigma_{\text {dos } \pm}$ were reported by Broch et al. in gate-doped DPP copolymer thin films (56-71meV). For instance, PDPP-3T is characterized by $\sigma_{\text {dos } \pm}=71 \mathrm{meV}$.

The values determined from our doping studies are of the same order of magnitude along the chain direction, albeit slightly larger in average to those obtained for other DPP-polymers. The larger $\sigma_{\text {dos }}$ in the doped films of this study may be related to the increased disorder generated by the $\mathrm{FeCl}_{3}$ dopant intercalation in the ordered domains of PDPP-5T as compared to pristine undoped films. This would be consistent with the TEM observations showing increased disorder in the thin films upon increasing concentration of $\mathrm{FeCl}_{3}$. This is also consistent with the results of Abutaha et al. who showed a link between paracrystallinity i.e. the degree of stacking disorder and the width of

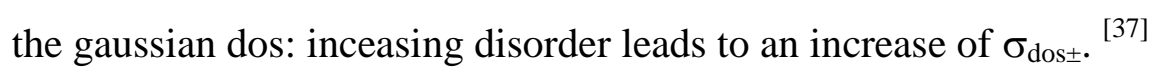

In contrast to PDPP-5T, $\mathrm{C}_{12}$-PBTTT doped with $\mathrm{FeCl}_{3}$ does not show a polarity switch even though a high doping level similar to that observed in the present case was reached. ${ }^{[10]}$ One reason for the 
polarity switch is that PDPP-based copolymers are intrinsically ambipolar with relatively high electron and hole mobilities in excess of $1 \mathrm{~cm}^{2} / \mathrm{V} \cdot \mathrm{s}$. Moreover, using Field-effect gated transistors, it has been shown that electrons and holes exhibit similar Seebeck coefficients. ${ }^{[36]}$ This was interpreted in a way that electrons and holes experience similar energetic landscapes. The present study on $\mathrm{FeCl}_{3}$-doped films demonstrates some imbalance between electron and holes with a larger energetic disorder for electrons.

\section{b) Scaling between thermopower and charge conductivity.}

Furthermore, the Fig. 6.b also displays the behavior expected according to the scaling relationship $\alpha \propto \sigma^{-1 / s}$ with $\mathrm{s}=4$ which is followed by a wide variety of conducting polymers away from the maximum of the dos $(|\alpha| \neq 0) .{ }^{[33]}$ Interestingly, a good adequacy with the experimental data is clearly demonstrated in Fig. 6.b up to $\sigma / \sigma_{\mathrm{w}}<0.5$. Since this scaling relationship is constrained by monotonic dos following a power law energy dependence, it cannot suitably reproduce the polarity switch regime. Nevertheless, it has been demonstrated quite recently that the $s=4$ scaling is consistent with massless pseudo-relativistic quasiparticles, namely Dirac fermions, scattered by unscreened ionized impurities. Since these quasiparticles are characterized in 3D by a parabolic dos, it could be extended in the vicinity of the dos maximum with a modified parabolic dos as the one previously discussed. As a result, the complete simulated behavior of the conductivity as a function of the thermopower should be given in Fig. $6 . b$ by the $s=4$ simulation up to nearly $\sigma / \sigma_{\mathrm{w}} \sim 0.3$ and then by the modified parabolic dos prediction in order to account for the maximum of the dos. This combination leads therefore to an agreement with the experimental data in the Fig. 6.b as good as the one observed for the Gaussian dos. So, in order to closer inspect these two descriptions with respect to the data, they can be plotted in a more conventional way, namely by showing the thermopower as a function of the electrical conductivity in double Log scale as in the Fig. 7. 

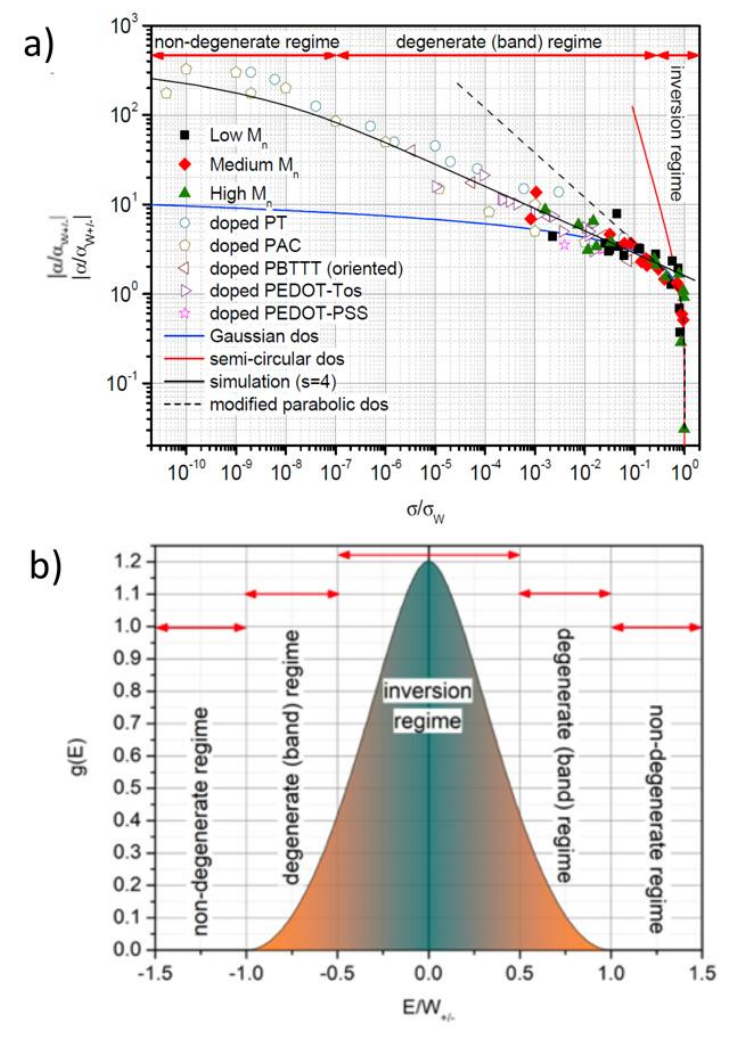

Figure 7. a) Scaling plot of the thermopower as a function of the electrical conductivity in double Log scale. Note that the non-oriented and oriented samples are here represented with the same full symbols in contrast to the Fig. 6.b for a sake of clarity. Both quantities are normalized as in the Fig. $6 . b$ and compared with data measured in other conducting polymers as already reported [33]. The latter are normalized with $\sigma_{W}=10^{4} \mathrm{~S} / \mathrm{cm}$ and $\alpha_{W}=4 \mu V / K$ for the PT, PAC, PEDOT-Tos and PEDOT-PSS while the higher value, $\sigma_{W}=310^{6} \mathrm{~S} / \mathrm{cm}$, is used for the oriented PBTTT due to its exceptionally high conductivity values. These data are compared with the theoretical predictions originating from the Gaussian, semi-circular, modified parabolic dos and the simulation leading to the scaling $\alpha \propto \sigma^{-1 / s}$ with $s=4$ in the degenerate regime [33]. The latter simulation helps describe the non-degenerate regime reached when $\sigma$ is very low and when the scaling relationship is no longer a power law but becomes logarithmic. Note that the simulation line intercepts the dashed one around $\sigma / \sigma_{W^{\sim}} 0.3$ and the combination of both predictions describes then the overall data including the inversion regime associated to the polarity switch. b) Modified parabolic dos as a function of energy in reduced unit. The energy extension of the three regimes of interest is shown according to the dos and the location of the Fermi level determines the transport regime as 
explained in the text. One may emphasize that the inversion regime is also degenerate but more specifically governed the dos curvature change.

In contrast to the Fig. 6.b, the representation in Figure 7.a mainly focuses on the regime away from the maximum of the dos which appears essentially as a vertical line indicating the polarity switch referred as the inversion regime. It turns out that when $\sigma / \sigma_{\mathrm{W}}<0.1$ the experimental data follow quite well the scaling behavior $\alpha \propto \sigma^{-1 / s}$ with $\mathrm{s}=4$ observed in many conducting polymers such as those reported in the Fig. 7.a. This agreement is checked over more than two decades and the data are successfully compared with the ones measured in other conducting polymers when using only one set of normalization constants for doped polythiophene (PT) and polyacethylene, (PAC), poly(3,4- ethylenedioxythiophene) doped with tosylate ions (PEDOT-Tos) or polystyrene sulfonate (PEDOT-PSS), with the exception of the oriented PBTTT which requires much higher $\sigma_{\mathrm{W}}$ due to its exceptionally high conductivity values ([33] and references therein).

A clear departure from the Gaussian behavior can be seen when $\sigma / \sigma_{\mathrm{w}}<10^{-2}$ suggesting then that the Gaussian dos is a good approximation but limited over a restricted range. One may assume that its partial success originates from its curvature similar to the one of the modified parabolic dos. It also appears as in the Fig. 7 that when the prediction of the modified parabolic dos (dashed line) departs from the experimental data around $\sigma / \sigma_{\mathrm{w}} \sim 0.3$, the agreement with the simulation ( $\mathrm{s}=4$ ) line becomes very good in the regime referred as degenerate. One can therefore interpret the overall behavior displayed in Fig. 8.a as it follows with the use of the density of states in the Fig. 7.b.

Considering an increasing doping, if the reduced Fermi level $\left(\mathrm{E}_{\mathrm{F}} / \mathrm{W}_{ \pm}\right)$falls outside the band, namely $\left|E_{F} / W_{ \pm}\right|>1$ in Fig. 10, the system is insulating and characterized by an energy gap. If $E_{F} / W_{ \pm}$is negative (respectively positive) the system is electron (hole)-like with a negative (positive) thermopower. This non-degenerate regime leads to a logarithmic correlation between the thermopower and the electrical conductivity as already discussed and observed experimentally 
$[25,28,33]$. By doping, the Fermi level can next decrease typically such as $0.5<\left|E_{\mathrm{F}} / \mathrm{W}_{ \pm}\right|<1$ and then the system becomes degenerate. In such a regime, the dos can be considered monotonic and varies as a power law (quadratic in the present case). In this degenerate regime, the full energy dependence needs to be taken into account in the aforementioned transport integrals in order to calculate the electrical conductivity and the thermopower. As already demonstrated, ${ }^{[25]}$ this regime is characterized by a power law scaling relationship such as $\alpha \propto \sigma^{-1 / s}$ with the exponent s which is the sum of the exponents of the energy dependence of the relaxation time, the charge carrier's velocity and the density of states. ${ }^{[33]}$ If the charge carriers are 3D Dirac fermions, the velocity is constant, the dos is parabolic and the relaxation time due to unscreened ionized impurities scattering is also quadratic in energy leading thus to the scaling exponent $s=4$ in agreement with the experimental behavior in the Fig. 7. a over more than 6 decades. ${ }^{[33]}$ Obviously, if it is chemically possible to further increase the doping, this regime is expected to breakdown due to the finite energy bandwidth. It means that a real dos necessarily displays a maximum separating the electron side from the hole one. This occurs roughly in Fig. 7.b if $\left|\mathrm{E}_{\mathrm{F}} / \mathrm{W}_{ \pm}\right|<0.5$, where the dos curvature change dominates over the other energy dependence in transport integrals. This explains why the approximation realized here with a constant relaxation time is suitable in order to describe the polarity switch in the inversion regime in Fig. 6.b and 7.a.

Finally, one may emphasize that the agreement between the Gaussian dos prediction and the experimental data in the Fig. 7.a could be likely slightly improved by using a more realistic relaxation time that takes into account an energy dependence associated to a scattering mechanism. Nevertheless, it will not allow to describe a power law scaling relationship between the thermopower and the electrical conductivity over an extended range due to the exponential dos. Therefore, our analysis demonstrates that the doping induced thermopower sign change is mainly governed by the filling of the dos and that the complete description of the different transport regimes requires very likely dos as a power law in order to be consistent with the scaling relationship observed in the degenerate regime by many authors. 


\section{c) Power factor versus conductivity scaling.}

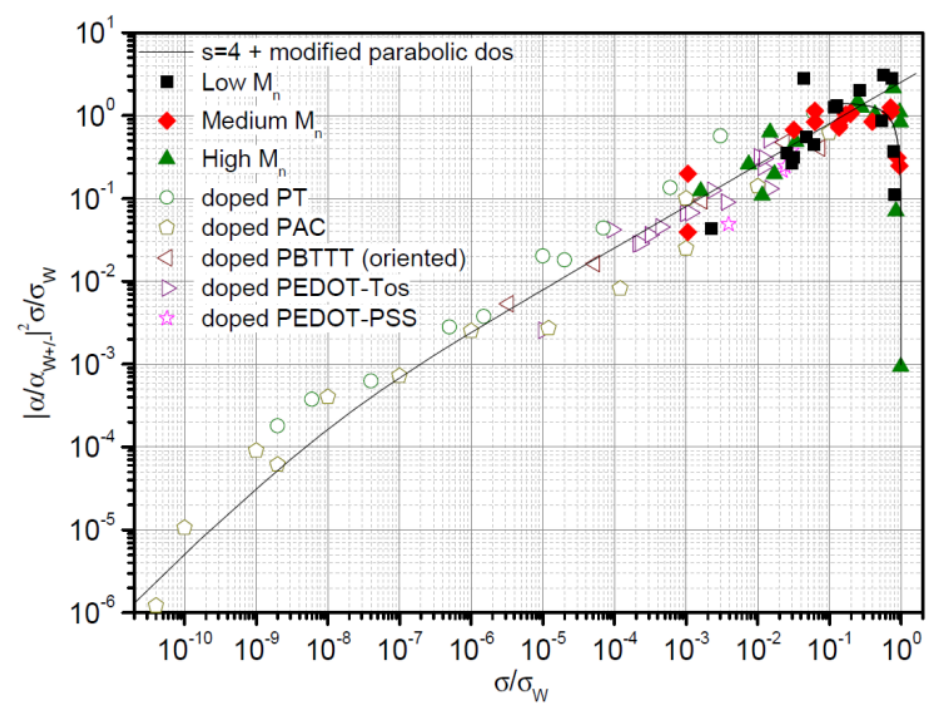

Figure 8. Normalized power factor as a function of the electrical conductivity as $\sigma / \sigma_{\mathrm{W}}$ in the investigated compounds (low, medium and high $\mathrm{M}_{\mathrm{n}}$ ) and in various conducting polymers as already reported [33].

From a more technological point of view, the best thermoelectric efficiency is given by the maximum of the figure of merit $\mathrm{Z}=\alpha^{2} \sigma / \kappa$ with the thermal conductivity $\kappa$. Therefore, the so-called power factor $\alpha^{2} \sigma$ provides an insight towards this maximum which is currently widely investigated in conducting polymers. ${ }^{[38]}$ By plotting in the Fig. 8 the normalized power factor as a function of the electrical conductivity versus $\sigma / \sigma_{\mathrm{W}}$ in the investigated compounds (low, medium and high $\mathrm{M}_{\mathrm{n}}$ ) and in various conducting polymers as already reported, ${ }^{[33]}$ the PF shows a characteristic maximum in the regime of high conductivity. More precisely, it is roughly located in the crossover region between the so-called band and the inversion regimes. It follows that this maximum appears in the degenerate regime at the end of the scaling behavior between thermopower and electrical conductivity when the dos no longer varies as a well-defined power law. Thus, the associated values of conductivity $\sigma / \sigma_{\mathrm{W}}$ which lie between 0.3 and 0.5 correspond also to $\mathrm{g}_{\mathrm{p}}\left(\mathrm{E}_{\mathrm{F}}\right) / \mathrm{g}_{\mathrm{p}, \mathrm{W}}$ and can be 
related to the doping by integration of the dos. This suggests that doped polymer semiconductors can show a maximum of the power factor for a doping level around $20 \%$, for both electron or hole carriers. This result seems consistent with the usually observed behavior in thermoelectric materials for which the maximum efficiency occurs in the heavily doped regimes and should help in designing high performance polymer-based thermolelectric devices.

\section{Conclusion.}

Doping oriented films of a donor-acceptor (D-A) copolymer PDPP-5T with $\mathrm{FeCl}_{3}$ produces highly conductive thin films with a charge conductivity up to $327 \mathrm{~S} / \mathrm{cm}$ along the chain direction. Polarity switching from p-type to n-type is observed with increasing doping concentration of $\mathrm{FeCl}_{3}$. Preliminary results indicate that similar polarity switching is also observed for other alternated copolymers. We propose an analysis based on non-monotonic density of states to account for the main experimental trends and demonstrate that the polarity switch is governed by the filling of a non-monotonic dos, with either gaussian or modified parabolic form, and that it is doping induced. The influence of the curvature of the density of states is discussed showing that the dos can be satisfactorily described by a gaussian form at low energy whose width could be determined. The polarity switching in PDPP-5T depends on the molecular weight $\mathrm{M}_{\mathrm{n}}$ of the polymer and shifts to higher $\mathrm{FeCl}_{3}$ concentrations with increasing $\mathrm{M}_{\mathrm{n}}$. This behavior is attributed to the change of the width of the density of states with $\mathrm{M}_{\mathrm{n}}$. Incidentally, the strategy of band filling of a p-type semiconductor by adjusting the doping level is an elegant method to probe the analytical form of the dos and extract its characteristic width for doped polymer semiconductors. Moreover, the combination of polarity switching and alignment is a mean to produce n-type conducting polymers with enhanced power factors up to $10 \mu \mathrm{W} \cdot \mathrm{K}^{-2} \cdot \mathrm{m}^{-1}$.

Acknowledgments. We thank the Agence Nationale de la Recherche for financial support through project ANR -17-CE05-0012 and CNRS through PEPS grant Thermobody. M. Schmutz and C. 
Blanck are acknowledged for technical support in TEM. L. Biniek is gratefully acknowledged for assistance in polymer film alignment.

\section{Supporting Information}

Polarized UV-vis-NIR spectra of pristine PDPP-5T films oriented by rubbing for different molecular weights. Electron diffraction pattern of low-M $\mathrm{M}_{\mathrm{n}}$ PDP-5T in pristine and doped form. Synthesis of PDPP-5T,

\section{Conflict of interest.}

The authors declare no conflicts of interests.

\section{Experimental section.}

1) Materials.

The details of the synthesis of the polymers are given in the SI. In brief, the DPP-based monomer M1 was synthesized in three steps with an overall yield of $20 \%$ according to modified literature procedures [39],[40],[41]. Poly[[2,5-di(2-octyldodecyl)pyrrolo[3,4-c]-pyrrole-1,4(2H,5H)-dione3,6-diyl]-alt-[2,2':5',2":5",2"':5"',2'"'-Quinquethiophene]-5,5”-diyl]] was prepared from dibromoDPP derivative (M1) and 5,5"-bis(trimethylstannyl)-2,2':5',2"-terthiophene (M2) by using Stille coupling polycondensation modified protocols [40],[42], following scheme ESI1. All polymers were obtained as blue or dark blue solids after careful purification of the precipitates into methanol containing concentrated hydrochloric acid and additional Soxlet extraction with methanol, hexane, chloroform and chlorobenzene. The three polymers show good solubility and can be dissolved in odichlorobenzene. Molecular weights of all polymers were evaluated by gel permeation chromatography (GPC) with 1,2,4-trichorobenzene at $150^{\circ} \mathrm{C}$ as eluent. 
Table 2. SEC measurements and molar extinction coefficient of polymers. ( $\varepsilon$ was calculated from Mp values)

\begin{tabular}{|c|c|c|c|c|c|c|}
\hline \multirow[b]{2}{*}{ Polymers } & \multirow[b]{2}{*}{ Yield $\%$} & \multicolumn{4}{|c|}{ SEC } & \multirow[b]{2}{*}{$\varepsilon\left[\mathrm{L} \cdot \mathrm{mol}^{-1} \cdot \mathrm{cm}^{-1}\right.$} \\
\hline & & $\begin{array}{c}M_{p} \\
{[\mathrm{Da}]}\end{array}$ & $\begin{array}{c}M_{w} \\
{[\mathrm{Da}]}\end{array}$ & $\begin{array}{c}M_{n} \\
{[\mathrm{Da}]}\end{array}$ & $P D I$ & \\
\hline Low- $\mathrm{M}_{\mathrm{n}}$ & 60 & 6434 & 14280 & 7351 & 1.9 & 291 \\
\hline $\begin{array}{c}\text { Medium- } \\
\mathrm{M}_{\mathrm{n}}\end{array}$ & 74.9 & 37981 & 50011 & 11246 & 4.4 & 388 \\
\hline High- $\mathrm{M}_{\mathrm{n}}$ & 16.1 & 40334 & 46242 & 16897 & 2,7 & 320 \\
\hline
\end{tabular}

\section{2) Orientation of thin films.}

The preparation of oriented PDPP-5T films by high-temperature rubbing followed the protocol described in previous publications. ${ }^{[19,20]}$ PDPP-5T films were prepared by doctor blading a hot polymer solution $(10 \mathrm{mg} / \mathrm{ml})$ in ortho-dichlorobenzene (oDCB) on NaPSS-coated glass substrates at $160^{\circ} \mathrm{C}$. The NaPSS films are spin coated $(3000 \mathrm{rpm}, 10 \mathrm{mg} / \mathrm{ml} \mathrm{aq})$ on pre-cleaned glass substrates and serve as sacrificial substrates to float the rubbed films on the pre-patterned devices for Conductivity/Seebeck measurements or on copper grids for TEM analysis. High temperature rubbing was performed by using a homemade set up consisting of a translating hot plate on which the sample is fixed and a rotating cylinder covered with a microfiber cloth. The films were rubbed at an optimal rubbing temperature leading to the best alignment depending on the molecular weight of PDPP-5T $\left(200^{\circ} \mathrm{C}\right.$ for low- $\mathrm{M}_{\mathrm{n}}, 125^{\circ} \mathrm{C}$ for medium- $\mathrm{M}_{\mathrm{n}}$ and $60^{\circ} \mathrm{C}$ for high- $\left.\mathrm{M}_{\mathrm{n}}\right)$. The film thickness was determined using the absorbance of the films and a calibration of film thickness was obtained by AFM (multimode 8 Bruker, peak force tapping mode with ScanAsyst-Air quantilever (0.4N/m)).

\section{3) Doping protocol.}

All doping experiments were carried out in a glove box (Jacomex) with $\mathrm{P}_{\mathrm{O} 2}<1 \mathrm{ppm}$ and $\mathrm{P}_{\mathrm{H} 2 \mathrm{O}}<1$ ppm. $10 \mathrm{mM} \mathrm{FeCl}$ solutions were prepared by dissolving $8 \mathrm{mg}$ of $\mathrm{FeCl}_{3}$ in $5 \mathrm{ml}$ anhydrous nitromethane in a glass vial and subsequent dilution yielded the solutions with concentration in the 
range 0.1 - $5 \mathrm{~mm}$. Doping was done by dipping the oriented polymer film in the dopant solution for 30 s until no color change was any more visible.

\section{3) Thin film characterization.}

Structural analysis: TEM: The preparation of the samples for TEM analysis involved three steps: i) floating the oriented polymer films on distilled water and recover them on TEM copper grids, ii) coat the films with a thin amorphous carbon layer using an auto 306 Edwards evaporator and iii) dope the TEM grids directly in the glove box by dipping the grids in a $\mathrm{FeCl}_{3}$ /nitromethane solution of given concentration. After 15-30s, the grids were blotted with a piece of absorbing tissue to remove the excess of dopant solution. The samples were exposed to air for 10-30s just before introducing the sample holder into the TEM. TEM was performed in the bright field and diffraction modes using a CM12 Philips microscope at 120kV equipped with an MVIII (Soft Imaging System) Charge Coupled Device camera. Beam exposure was set to a minimum using the low dose system to avoid de-doping under the electron beam that is observed when the same zone is exposed for a prolonged period of time.

Polarized UV-Vis-NIR absorption: The orientation of the polymer films was probed by UV-VisNIR absorption (350-2500 nm) using a Cary 5000 spectrometer with polarized incident light and spectral resolution of $1 \mathrm{~nm}$. The UV-Vis-NIR spectra of the doped polymer film were measured parallel and perpendicular to the rubbing direction $\mathrm{R}$.

Electrical conductivity and Seebeck Coefficient: All devices were fabricated on glass substrates following the conditions described in references 8-10. 40 nm-thick gold contacts were prepared by thermal evaporation using appropriate shadow masks. The contact geometry used for the electrical conductivity and thermopower measurements is given in reference 8 . The oriented PDPP-5T films prepared by rubbing on NaPSS substrates were floated on distilled water and subsequently recovered on the devices with gold electrodes. Doping with $\mathrm{FeCl}_{3}$ was performed by dipping in the solutions of given concentration. Electrical conductivity was measured using the four-point-probe 
geometry and the procedure described in detail in reference 8. In brief, a Keithley 2634B sourcemeter and a Lab Assistant Semiprobe station in a Jacomex glovebox under $\mathrm{N}_{2}$ atmosphere allowed to measure both conductivity and Seebeck coefficients on the same devices. The resistivity $\rho$ was derived from the sheet resistance R following the equation $\rho=1.81 \mathrm{Rt}$ (consider reference 24 for the determination of the geometrical correction factor). The Seebeck coefficient $S$ were derived from the $\Delta \mathrm{V}=\mathrm{S} \Delta \mathrm{T}$ curves.

\section{References.}

(1) R. Kroon, D. A. Mengistie, D. Kiefer, J. Hynynen, J. D. Ryan, L. Yu, C. Müller, Chem. Soc. Rev. 2016, 45, 6147.

(2) O. Bubnova, and X. Crispin, Energy Environ. Sci. 2012, 5 (11), 9345-9362.

(3) O. Bubnova, Z. U. Khan, A. Malti, S. Braun, M. Fahlman, M. Berggren and X. Crispin, Nature Materials 2011, 10 (6), 429-433.

(4) G. Zuo, H. Abdalla and M. Kemerink, M. Advanced Electronic Materials 2019, 5 (11), 1800821.

(5) R. Kroon, D. Kiefer, D. Stegerer, L. Yu, M. Sommer, C. Müller, Advanced Materials 2017, 29, 1700930.

(6) J. Hynynen, D. Kiefer, L. Yu, R. Kroon, R. Munir, A. Amassian, M. Kemerink, C. Müller, Macromolecules 2017, 50, 8140.

(7) A. M. Glaudell, J. E. Cochran, S. N. Patel, M. L. Chabinyc, Advanced Energy Materials 5, 1401072.

(8) A. Hamidi-Sakr, L. Biniek, J.-L. Bantignies, D. Maurin, L. Herrmann, N. Leclerc, P. Leveque, V. Vijayakumar, N. Zimmermann and M. Brinkmann, Adv. Funct. Mater. 2017, 27 (25), 1700173.

(9) V. Vijayakumar, E. Zaborova, L. Biniek, H. Zeng, L. Herrmann, A. Carvalho, O. Boyron, N. Leclerc and M. Brinkmann, ACS Appl. Mater. Interfaces 2019, 11, 4942-4953.

(10) V. Vijayakumar, Y. Zhong, V. Untilova, M. Bahri, L. Herrmann, L. Biniek, N. Leclerc and M. Brinkmann, Adv. Energy Mater. 2019, 9 (24), 1900266.

(11) Y. M. Gross, D. Trefz, C. Dingler, D. Bauer, V. Vijayakumar, V. Untilova, L. Biniek, M. Brinkmann and S. Ludwigs, Chem. Mater. 2019, 31 (9), 3542-3555.

(12) K. Shi, F. Zhang, C.-A. Di, T.-W. Yan, Y. Zou, X. Zhou, D. Zhu, J.-Y. Wang, and J. Pei, J. Am. Chem. Soc. 2015, 137 (22), 6979-6982.

(13) J. Liu, G. Ye, B. Zee, J. van der; Dong, X. Qiu, Y. Liu, G. Portale, R. C. Chiechi, L. J. A. Koster, Adv. Mater. 2018, 30 (44), 1804290. 
(14) S. Hwang, W. J. Potscavage, Y. S. Yang, I. S. Park, T. Matsushima, C. Adachi, Phys. Chem. Chem. Phys. 2016, 18 (42), 29199-29207.

(15) F. S. Kim, X. Guo, M. D. Watson and S. A. Jenekhe, Adv. Mater. 2010, 22 (4), 478-482.

(16) I. H. Jung,C. T. Hong, U.-H. Lee, Y. H. Kang, K.-S. Jang and S. Y. Cho, Scientific Reports 2017, $7(1), 44704$.

(17) B. Yurash, D. X. Cao, V. V. Brus, D. Leifert, M. Wang, A. Dixon, M. Seifrid, A. E. Mansour, A. E., D. Lungwitz, T. Liu, P. J. Santiago, K. R. Graham, N. Koch, G. C. Bazan and T.-Q. Nguyen, Nature Materials 2019, 18 (12), 1327-1334.

(18) L. Biniek, S. Pouget, D. Djurado, E. Gonthier, K. Tremel, N. Kayunkid, E. Zaborova, N. Crespo-Monteiro, O. Boyron, N. Leclerc, S. Ludwigs and M. Brinkmann Macromolecules 2014, 47 (12), 3871-3879.

(19) Y. Zhong, L. Biniek, N. Leclerc, S. Ferry and M. Brinkmann, Macromolecules, 2018, 51, $4238-4249$.

(20) Y. Karpov, T. Erdmann, M. Stamm, U. Lappan, O. Guskova, M. Malanin, I. Raguzin, T. Beryozkina, V. Bakulev, F. Günther, S. Gemming, G. Seifert, M. Hambsch, S. Mannsfeld, B. Voit and A. Kiriy, Macromolecules, 2017, 50, 914-926.

(21) R. Ghosh, C. M. Pochas and F. C. Spano, J. Phys. Chem. C 2016, 120 (21), 11394-11406.

(22) R. Ghosh, A. R. Chew, J. Onorato, V. Pakhnyuk, C. K. Luscombe, A. Salleo and F. C. Spano, J. Phys. Chem. C 2018, 122 (31), 18048-18060.

(23) V. Untilova, T. Biskup, L. Biniek, V. Vijayakumar and M. Brinkmann, Macromolecules, 2020, 53, 2441.

(24) R. J. Kline, M. D. McGehee, E. N. Kadnikova, J. Liu, J. M. J. Fréchet, M. F. Toney, Macromolecules 2005, 38 (8), 3312-3319.

(25) S. D. Kang, and J. Snyder, Nat. Mater. 16, 252-257 (2017).

(26) J. M. Ziman, Electrons and Phonons (Oxford Univ. Press, 1960).

(27) N. W. Ashcroft, and N. D. Mermin, Solid State Physics (Saunders College, New York, 1976).

(28) N. F. Mott, and E. A. Davis, Electronic Processes in Non-Crystalline Materials 2nd edition (Oxford Univ. Press, 1979).

(29) H. Bässler, Phys. Stat. Sol. B 1993, 175, 15.

(30) G. Zuo, H. Abdalla, and M. Kemerink, Phys. Rev. B 2016, 93, 235203.

(31) H. Abdalla, G. Zuo, and M. Kemerink, Phys. Rev. B 2017, 96, 241202(R). 
(32) C. J. Boyle, M. Upadhyaya, P. Wang, L. A. Renna, M. Lu-Díaz, S. Pyo Jeong, N. HightHuf, L. Korugic-Karasz, M. D. Barnes, Z. Aksamija, D. Venkataraman, Nature Communications 2019, 10, 2827.

(33) M. Lepinoy, P. Limelette, B. Schmaltz, B. and F. Tran Van, Scientific Reports 2020, $10,8086$.

(34) S. Hood, N. Zarrabi, P. Meredith, I. Kassal and A. Armin, J. Phys. Chem. Lett. 2019, 10, 3863-3870.

(35) F. Maddalena, C. de Falco, M. Caironi and D. Natali, Org. Elect. 2015, 17, 304-318.

(36) K. Broch, D. Venkateshvaran, V. Lemaur, Y. Olivier, D. Beljonne, M. Zelazny, I. Nasrallah, D. J. Harkin, M. Statz, R. D. Pietro, A. J. Kronemeijer, H. Sirringhaus, Advanced Electronic Materials 2017, 3, 1700225.

(37) A. Abutaha, P. Kumar, E. Yildirim, W. Shi, S.-W. Yang, G. Wu and K. Hippalgaonkar, Nat. comm. 2020, 11, 1737.

(38) H. Tanaka, K. Kanahashi, N. Takekoshi, H. Mada, H. Ito, Y. Shimoi, H. Ohta, T. Takenobu, Sci Adv 2020, 6, eaay8065.

(39) L. Huo et al., "Bandgap and molecular level control of the low-bandgap polymers based on 3,6-dithiophen-2-yl-2,5-dihydropyrrolo[3,4-c]pyrrole-1,4-dione toward highly efficient polymer solar cells," Macromolecules, vol. 42, no. 17, pp. 6564-6571, 2009.

(40) Y. Li, S. P. Singh and P. Sonar, Adv. Mater. 2010, vol. 22, no. 43, 4862-4866.

(41) E. Zhou, Q. Wei, S. Yamakawa, Y. Zhang, K. Tajima, C. Yang, K. Hashimoto, Macromolecules 2010, 43, 821.

(42) R. Di Pietro, T. Erdmann, J. H. Carpenter, N. Wang, R. R. Shivhare, P. Formanek, C. Heintze, B. Voit, D. Neher, H. Ade, A. Kiriy, Chem. Mater. 2017, 29, 10220. 


\title{
Fabrication of oriented n-type thermoelectric polymers by polarity switching in a DPP-based Donor-Acceptor copolymer doped with a strong Lewis acid
}

\author{
Huiyan Zeng(1), Mohammed Mohammed (2), Viktoriia Untilova(1), Olivier Boyron (3), Nicolas \\ Berton (2), Patrice Limelette (4) and Bruno Schmaltz (2), Martin Brinkmann(1)
}

(1) Université de Strasbourg, CNRS, ICS UPR 22, F-67000 Strasbourg, France

(2) PS2M, Université de Tours, Parc de Grandmont, F-37200, Tours, France

(3) Lyon

(4) GREMAN, UMR 7347 CNRS-INSA-Université de Tours, Parc de Grandmont, F-37200 TOURS, FRANCE

Corresp. Authors :

Martin.brinkmann@ics-cnrs.unistra.fr

bruno.schmaltz@univ-tours.fr

patrice.limelette@univ-tours.fr 


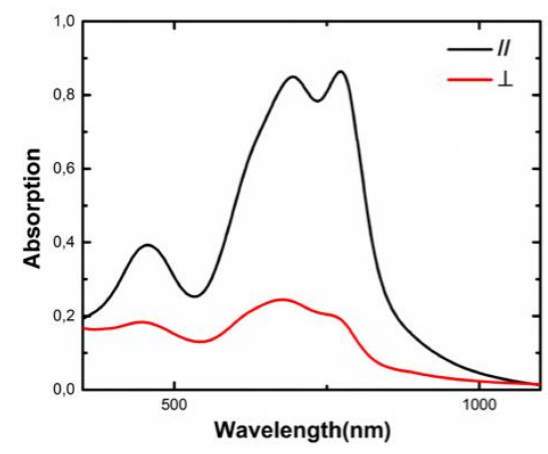

Low-Mn

$\mathrm{T}_{\mathrm{R}} 200^{\circ} \mathrm{C} \operatorname{Dr}(760 \mathrm{~nm})=3$

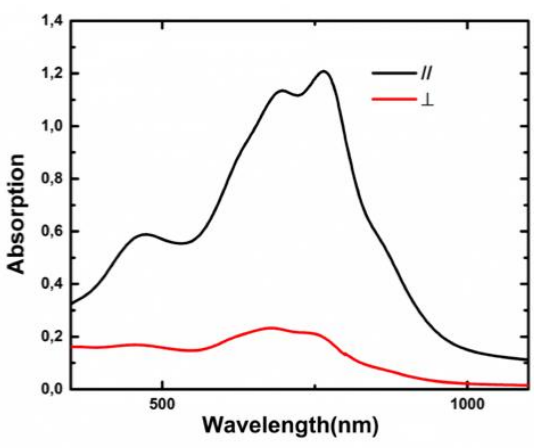

Medium-Mn

$\mathrm{T}_{\mathrm{R}} 125^{\circ} \mathrm{C} \operatorname{Dr}(760 \mathrm{~nm})=5.5$

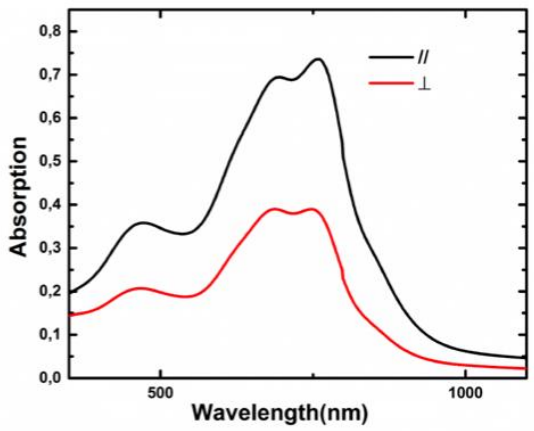

High-Mn

$\mathrm{T}_{\mathrm{R}} 200^{\circ} \mathrm{C} \operatorname{Dr}(760 \mathrm{~nm})=2.1$

Figure ESI1. Polarized UV-vis spectra of oriented thin films of PDPP-5T oriented by hightemperature rubbing for samples of different molecular weight.

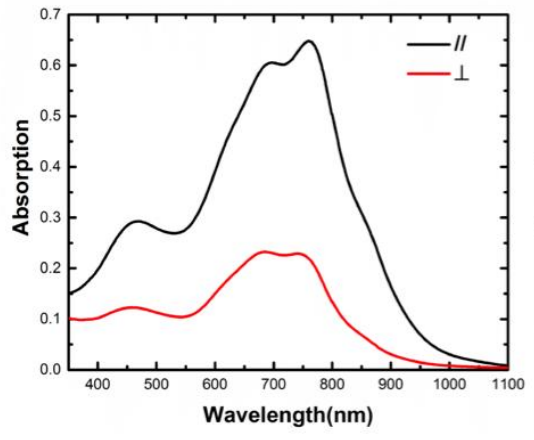

$T_{R} 80^{\circ} \mathrm{C} \operatorname{Dr}(760 \mathrm{~nm})=2.8$

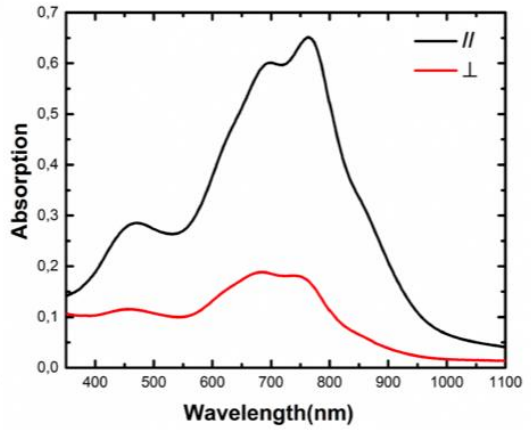

$\mathrm{T}_{\mathrm{R}} 100^{\circ} \mathrm{C} \operatorname{Dr}(760 \mathrm{~nm})=3.8$

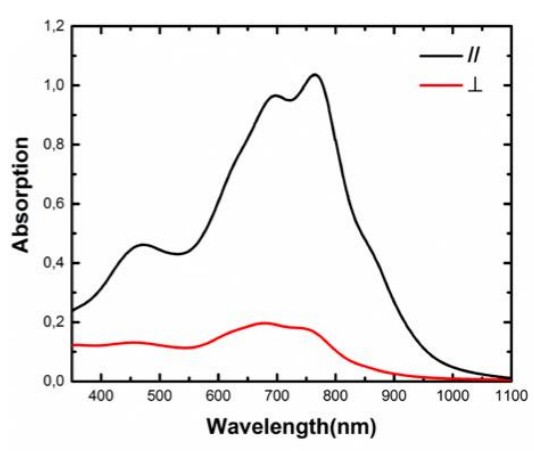

$\mathrm{T}_{\mathrm{R}} 125^{\circ} \mathrm{C} \operatorname{Dr}(760 \mathrm{~nm})=5.5$

Figure ESI2. Polarized UV-vis spectra of oriented thin films of Medium-Mn PDPP-5T oriented by high-temperature rubbing for samples of different rubbing temperatures $T_{R}$. A maximum dichroic ratio DR of 5.5 is obtained for $T_{R}=125^{\circ} \mathrm{C}$. 


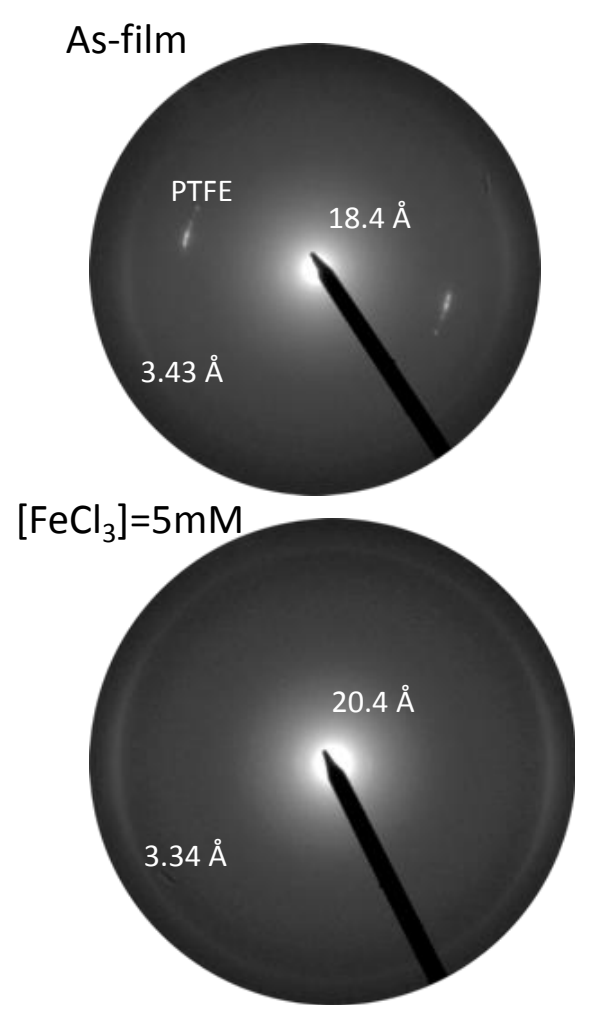

Figure ESI3. Change of the ED pattern in aligned PDPP-5T films $\left(l o w-M_{n}\right)$ from pristine to doped film $\left(\left[\mathrm{FeCl}_{3}\right]=5 \mathrm{mM}\right)$. 


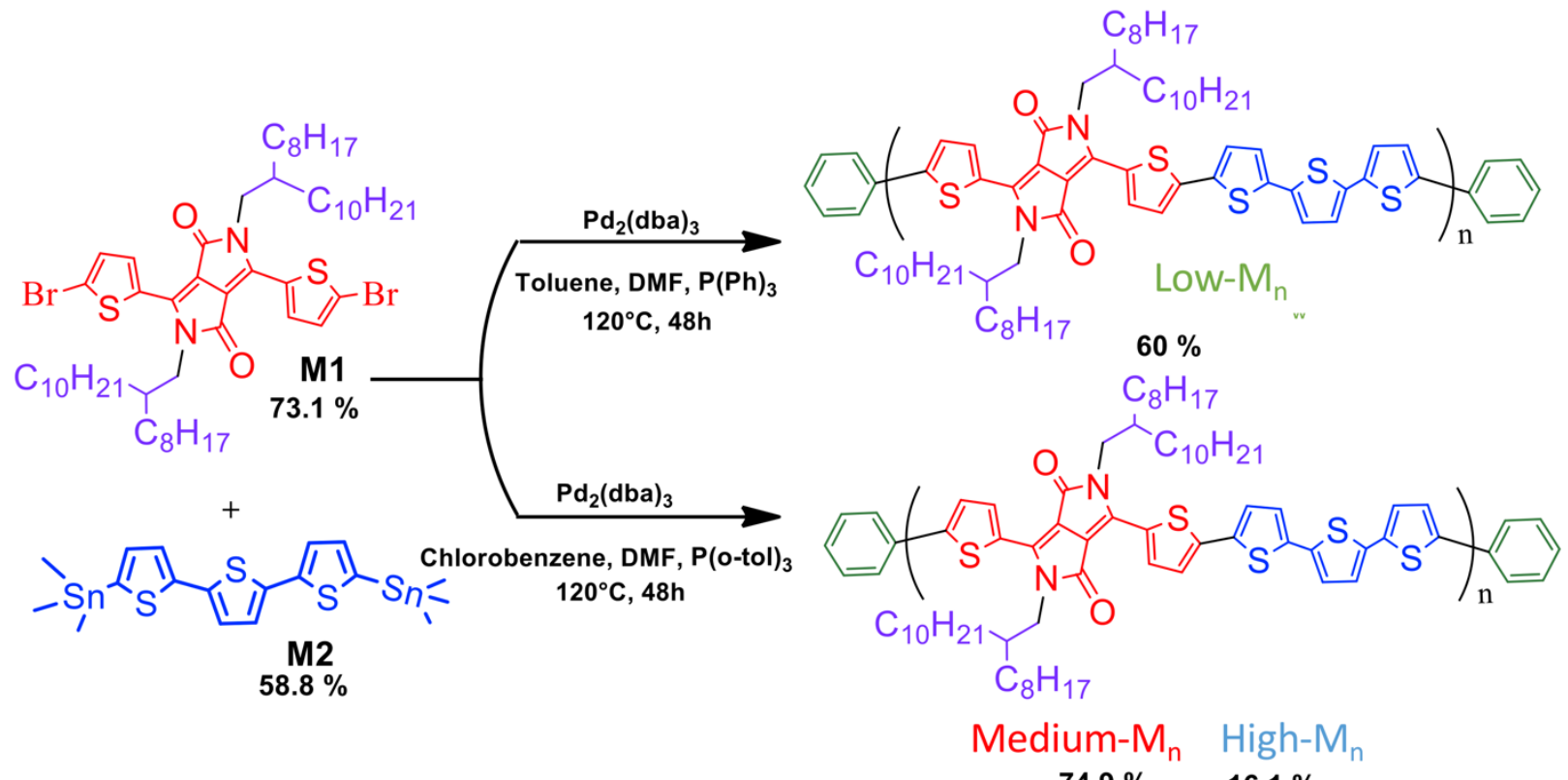

Figure ESI 4. Stille polymerization for the PDPP-5T polymers.

\section{Polymer synthesis.}

Materials: All chemicals were used without purification unless otherwise indicated. Potassium tert-butoxide, 2-thiophenecarbonitrile, t-amyl alcohol, dimethyl succinate, n-buthyllithium (BuLi, 1.6 M solution in hexane), trimethylstannyl chloride ( $\mathrm{TMSnCl}, 1 \mathrm{M}$ solution in THF), tris (dibenzylideneacetene) dipalladium(0), tri-phosphine, tri-o-tolylphosphine, bromobenzene, $\mathrm{N}$ Bromosuccinimide (NBS), $\mathrm{K}_{2} \mathrm{CO}_{3}$, anhydrous toluene, anhydrous chlorobenzene, anhydrous DMF, anhydrous THF and ethyl ether were purchased from Sigma Aldrich. Acetic acid, anhydrous acetonitrile, 2-octyldodecan-1-ol were purchased from Alfa Aesar. Magnesium sulfate $\mathrm{MgSO}_{4}$ was purchased from Carlo Erba. Tetrabutyl ammonium hexafluorophosphate $\left(\mathrm{TBAPF}_{6}\right)$ was purchased from TCI. Methanol, ethanol, chloroform, dichloromethane (DCM), hexane, cyclohexane, ethylacetate were purchased from Sigma Aldrich, Alfa Aesar or Fluka.

Characterization methods: ${ }^{1} \mathrm{H}-\mathrm{NMR}$ and ${ }^{13} \mathrm{C}-\mathrm{NMR}$ spectra were recorded on a Bruker Avance $300(300 \mathrm{MHz})$ spectrometer. Infrared analysis has been performed on a Perkin-Elmer Spectrum- 
One FTIR spectrometer in $\mathrm{KBr}$ pellets. The molecular weight of the polymers were determined by high temperature Gel permeation chromatography (GPC-HT), 1,2,4-trichlorobenzene (TCB ) was using as the eluent at $150^{\circ} \mathrm{C}$, and polystyrene (PSS) for calibration. UV-visible spectra on a Jasco V-670 spectrometer. Thermogravimetric analysis (TGA) was fulfilled using a Perkin Elmer STA 6000 at a heating rate of $10^{\circ} \mathrm{C} / \mathrm{min}$ under $\mathrm{N}_{2}$. Differential scanning calorimetry (DSC) measurements were performed on Perkin-Elmer DSC-400 (heating/cooling rate 10 and $20^{\circ} \mathrm{C} / \mathrm{min}$ ) under $\mathrm{N}_{2}$. Cyclic voltammetry (CV) was performed using a Biologic Applied Research MPG2 multi-channel potentiostat, the $\mathrm{CV}$ experiments were performed on the polymers thin films that were coated onto the working electrode (platinum disk) into solution of $0.1 \mathrm{M}$ tetraethylammonium tetrafluoroborate $\left(\mathrm{TEABF}_{4}\right)$ in anhydrous acetonitrile $\left(\mathrm{CH}_{3} \mathrm{CN}\right)$ as conducting electrolyte, at a scan rate of $50 \mathrm{mV} / \mathrm{s}$, at room temperature (under nitrogen) in a three-electrode cell using platinum disk and wire as working and counter electrode, respectively. A silver wire was used as a pseudoreference electrode and ferrocene was used as internal reference. The $E_{\text {HOMO }}$ and $E_{\mathrm{LUMO}}$ of the polymers were calculated from the oxidation onset potentials relative to ferrocene as an internal standard $\mathrm{Fc} / \mathrm{Fc}^{+}$is $-5.1 \mathrm{eV}$. versus vacuum, [1] with the following equations:

$$
\begin{gathered}
E_{\mathrm{HOMO}}=-\left[E_{\text {ox }}^{\text {onset }}-E_{F c / F c+}^{\text {onset }}\right]-5.1 \mathrm{eV} .(1) \\
E_{\text {LUMO }}=E_{\text {HOMO }}-E_{g}^{\text {opt. }} \mathrm{eV} . \text { (2) }
\end{gathered}
$$

where $E_{o x}^{\text {onset }}$ and $\boldsymbol{E}_{\boldsymbol{F} \boldsymbol{c} / \boldsymbol{F} \boldsymbol{c}+}^{\text {onset }}$ are respectively the onset oxidation potentials for the polymer films and the ferrocene against the $\mathrm{Ag}$ reference electrode, while the value of $-5.1 \mathrm{eV}$. is its $\mathrm{HOMO}$ energy.

\section{Synthesis of monomers:}

Figure ESI5. Synthesis of monomers M1 and M2. 


\section{3,6-Dithiophen-2-yl-2,5-dihydropyrrolo[3,4-c]pyrrole-1,4-dione (1)}

1 was prepared following the procedure described for [2]. Potassium tert-butylate $(8 \mathrm{~g}, 71.6 \mathrm{mmol}$, 1.2 eq.) was added into a $250 \mathrm{~mL}$ three-neck flask with argon protection. A solution of 2thiophenecarbonitrile $(6.54 \mathrm{~g}, 60 \mathrm{mmol}, 1 \mathrm{eq})$ in $50 \mathrm{ml}$ of t-amyl alcohol is injected in one portion. Then, the mixture was warmed to $100-110{ }^{\circ} \mathrm{C}$ and a solution of dimethyl succinate $(2.92 \mathrm{~g}, 20$ mmol, $2.61 \mathrm{ml}, 0.33$ eq.) in $16 \mathrm{ml}$ of t-amyl alcohol was added slowly. The methanol by-product was removed by distillation and the reaction was refluxed followed by TLC until the starting material had disappeared ( 2 hours). Then, the mixture was cooled down to $65^{\circ} \mathrm{C}$, diluted with 100 $\mathrm{ml}$ of methanol and then the solution was neutralized with $10 \%$ vol. acetic acid. and bring to reflux for 10 additional minutes. Then the solution was filtered and the black solid, which precipitate on Buchner, was rinsed twice with hot methanol and water. The solid was dried under vacuum to give product $\mathbf{1}$ as a dark red solid which can be used directly without further purification (3.69 g, 61\% yield). ${ }^{1} \mathrm{H}-\mathrm{NMR}\left(300 \mathrm{MHz}, \mathrm{DMSO}-\mathrm{d}_{6}\right): \delta(\mathrm{ppm}) 7.31\left(\mathrm{t}, 2 \mathrm{H}, H-4\right.$ ); 7.96 (d, 2H, J=4.8 Hz, $\left.H-3^{\prime}\right)$; $8.23(\mathrm{~d}, 2 \mathrm{H}, \mathrm{J}=3.9 \mathrm{~Hz}, H-5$ ) $) 11.26$ (s, $2 \mathrm{H}, N-H) ;{ }^{13} \mathrm{C}-\mathrm{NMR}\left(75 \mathrm{MHz}, \mathrm{DMSO}-\mathrm{d}_{6}\right): \delta(\mathrm{ppm}) 162.1$, 136.6, 133.2, 131.7, 131.3, 129.2, 109.

\section{Synthesis of 2-octyldodecyl bromide (2)}

2-octyldodecyl bromide (2) was prepared by the adaptation of the method describes in ref [3]. 2octyldodecan-1-ol (of $4 \mathrm{~g}, 13.4 \mathrm{mmol}$ ) and (5.6 g, $21.4 \mathrm{mmol}$ ) of triphenylphosphine were dissolved in dichloromethane $(9 \mathrm{~mL})$ and cooled to $0{ }^{\circ} \mathrm{C}$. N-Bromosuccinimide $(3.6 \mathrm{~g}, 20.1 \mathrm{~mol})$ was added slowly to this mixture, and the reaction mixture was stirred at room temperature for $12 \mathrm{~h}$. The solvent was removed, and the residue was dispersed in hexane (a large amount of an orange solid did not dissolve), and the resulting precipitate $\left(\mathrm{Ph}_{3} \mathrm{PO}\right)$ was filtered and discarded. After the removal of the solvent, the filtrate was purified by column chromatography on silica gel using hexane as the eluent to give the pure product (2-octyldodecyl bromide) as a colorless oil ( $4.4 \mathrm{~g}$, 
91.2\% yield). ${ }^{1} \mathrm{H}-\mathrm{NMR}\left(300 \mathrm{MHz}, \mathrm{CDCl}_{3}\right): \delta$ (ppm) 3,44 (d, 2H, Br- $\mathrm{CH}_{2}$ ), 1,59 (m, IH), 1,27 (m, $\left.32 \mathrm{H},-\mathrm{CH}_{2}\right), 0,88\left(\mathrm{t}, 6 \mathrm{H},-\mathrm{CH}_{3}\right)$.

\section{Synthesis of 2,5-Octyldodecxyl-3,6-dithiophen-2-ylpyrrolo[3,4-c]pyrrole-1,4-dione (3)}

3 was prepared following a modified procedure described in reference [4]. In a dry three-neck round bottom flask, 1 ( $1 \mathrm{~g}, 3.3 \mathrm{mmol}, 1$ eq.) and anhydrous $\mathrm{K}_{2} \mathrm{CO}_{3}(1.8 \mathrm{~g}, 13.3 \mathrm{mmol}$, 4 eq.) were dissolved in $50 \mathrm{ml}$ of anhydrous DMF and heated at $130^{\circ} \mathrm{C}$ under argon for $1 \mathrm{~h}$. 2-Octyl-1dodecylbromide (5.5 g, $15.3 \mathrm{mmol}, 4.84 \mathrm{ml}, 4,6$ eq.) was then added dropwise during $20 \mathrm{~min}$. The reaction mixture was stirred and heated overnight at $140^{\circ} \mathrm{C}$. The reaction mixture was allowed to cool down to room temperature, poured into $100 \mathrm{~mL}$ of ice. The product was extracted with dichloromethane, washed with water, brine and dried over $\mathrm{MgSO}_{4}$. After distillation of the solvent, the crude product was further purified using a column chromatography on silica gel with cyclohexane/ethylacetate $(8 / 2)$ as eluent. The product is a purple solid $(1.2 \mathrm{~g}$, yield $43 \%) .{ }^{1} \mathrm{H}-\mathrm{NMR}$ $\left(300 \mathrm{MHz}, \mathrm{CDCl}_{3}\right): \delta(\mathrm{ppm})$ 8.87(d, $\left.2 \mathrm{H}, \mathrm{Th}\right), 7.52(\mathrm{~d}, 2 \mathrm{H}, \mathrm{Th}), 7.26(\mathrm{~d}, 2 \mathrm{H}, \mathrm{Th}), 4.00(\mathrm{~d}, 4 \mathrm{H}, \mathrm{N}-$ $\left.\mathrm{CH}_{2}\right), 1.89(\mathrm{~s}, 2 \mathrm{H}), 1.13-1.45\left(\mathrm{~m}, 64 \mathrm{H},-\mathrm{CH}_{2}\right), 0.87\left(\mathrm{t}, 12 \mathrm{H},-\mathrm{CH}_{3}\right) ;{ }^{13} \mathrm{C}-\mathrm{NMR}\left(75 \mathrm{MHz}, \mathrm{CDCl}_{3}\right)$ : $\delta(\mathrm{ppm}) 161.7,140.4,135.2,130.4,129.8,128.4,107.9,65.7,46.2,40.5,37.7,31.9,30.0,29.6,29.3$, $26.2,22.6,14.1$.

\section{Synthesis of 2,5-Octyldodecxyl-3,6-bis(5-bromothiophen-2-yl)pyrrolo[3,4-c]-pyrrole-1,4-dione (M1)}

M1 was prepared following a modified procedure described reference [5]. In a $250 \mathrm{~mL}$ roundbottomed flask, $3(0.5 \mathrm{~g}, 0.58 \mathrm{mmol}, 1 \mathrm{eq})$ was dissolved in $36 \mathrm{~mL}$ of $\mathrm{CHCl}_{3}$. The flask was cooled to $0^{\circ} \mathrm{C}$ under argon, and covered with aluminum foil. $\mathrm{N}$-Bromosuccinimide $(0.21 \mathrm{~g}, 1.19 \mathrm{mmol}$, 2.05 eq.) was added in portions, and the reaction was stirred for $16 \mathrm{~h}$ at room temperature. Then, the solvent was removed under reduced pressure. The residue was dissolved in DCM and extracted with water, brine and dried over $\mathrm{MgSO}_{4}$. The product is purified on silica gel with cyclohexane/EtOAc (8/2) as eluent to afford the product as a dark purple solid $(0.43 \mathrm{~g}, 73 \%$ yield).

${ }^{1} \mathrm{H} \mathrm{NMR}\left(\mathrm{CDCl}_{3}, 300 \mathrm{MHz}\right): \delta(\mathrm{ppm}) 8.62$ (d, 2H, Th), 7.21 (d, 2H, Th), $3.91\left(\mathrm{t}, 4 \mathrm{H}, \mathrm{N}-\mathrm{CH}_{2}\right), 1.88$ 
(b, 2H, $-\mathrm{CH}), 1.33-1.22\left(\mathrm{~m}, 64 \mathrm{H},-\mathrm{CH}_{2}\right), 0.89-0.86\left(\mathrm{t}, 12 \mathrm{H},-\mathrm{CH}_{3}\right) ;{ }^{13} \mathrm{C}-\mathrm{NMR}\left(75 \mathrm{MHz}, \mathrm{CDCl}_{3}\right): \delta$ (ppm) 161.4,139.4, 135.3, 131.4, 131.1, 118.9, 108, 86.3, 46.3, 37, 7, 31.9, 29.9, 29.6, 29.4, 26.2, 22.7, 22.4, 14.1, 14.1 .

\section{Synthesis of 5,5'-bis(trimethylstannyl)-2,2':5',2"-terthiophene (M2).}

M2 was prepared following a modified procedure described in reference [6]. A dry Schlenk tube was charged with 2,2': $: 5^{\prime}, 2^{\prime}{ }^{\prime}$-terthiophene (0.250 g, 1 mmol, 1eq.) and dry THF (2.8 mL). The solution was cooled to $-78{ }^{\circ} \mathrm{C}$ and $\mathrm{n}$-BuLi $(1.9 \mathrm{~mL}, 3 \mathrm{mmol}, 3$ eq.) was added dropwise to the schlenk tube. The solution was stirred for $2 \mathrm{~h}$ at room temperature. Then, the reaction mixture was cooled to $-78{ }^{\circ} \mathrm{C}$ and trimethyltin chloride $(3.5 \mathrm{~mL}, 3.54 \mathrm{mmol}, 3.5$ eq.) was added dropwise to the Schlenk tube. The reaction mixture was allowed to warm to room temperature and stirred overnight. The reaction was quenched with water and extracted with DCM. The organic layer was washed with brine. The green organic layer was then dried over $\mathrm{MgSO}_{4}$ and the solvent was removed under vacuum. The crude product was recrystallized twice from methanol to give light green crystal $(0.34$ g, 59\% yield). ${ }^{1} \mathrm{H}-\mathrm{NMR}\left(300 \mathrm{MHz}, \mathrm{CDCl}_{3}\right): \delta(\mathrm{ppm}) 0.39\left(\mathrm{~s}, 18 \mathrm{H}, \mathrm{CH}_{3}\right), 7.07(\mathrm{~d}, 4 \mathrm{H}, \mathrm{Th}), 7.27(\mathrm{~d}$, $2 \mathrm{H}, \mathrm{Th}) ;{ }^{13} \mathrm{C}-\mathrm{NMR}\left(75 \mathrm{MHz}, \mathrm{CDCl}_{3}\right): \delta(\mathrm{ppm}) 142.9,137.7,136.3,036.1,124.9,124.3,8.1$.

\section{Synthesis of polymers}

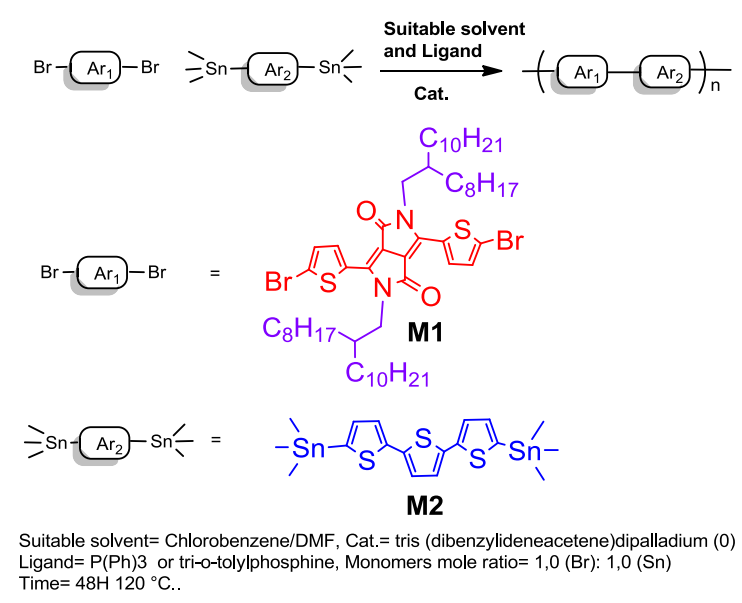

Figure ESI6. General synthetic route of polymers via Stille coupling method. 
The DPP copolymers were synthesized through a combination of donor-acceptor moieties by Stille cross-coupling polymerization as shown in Figure ESI6.

Poly[[2,5-di(2-octyldodecyl)pyrrolo[3,4-c]-pyrrole-1,4(2H,5H)-dione-3,6-diyl]-alt-[2,2':5',2':5", 2 "':5"',2"'"-Quinquethiophene]-5,5”-diyl]] (low-M, PDPP-5T)

The polymer was synthesized according to the literature [7,4]. M1 (163 mg, 0,16 mmol, 1eq.), M2 (92 $\mathrm{mg}, 0,16 \mathrm{mmol}$, 1eq.) were dissolved in $4 \mathrm{~mL}$ of anhydrous toluene and $0.4 \mathrm{ml}$ of anhydrous DMF. Then, tris(dibenzylideneacetene)dipalladium(0) $(4.4 \mathrm{mg}, 4.8 \mu \mathrm{mol}, 0.03$ eq. $)$ and triphenylphosphine ( $5 \mathrm{mg}, 19 \mu \mathrm{mol}, 0.118$ eq.) were added. The mixture was stirred at $120^{\circ} \mathrm{C}$ for 48 $\mathrm{h}$ followed by addition of the end-cappers. The mixture was stirred at $120^{\circ} \mathrm{C}$ for 6 additional hours. The mixture was precipitated in methanol and the solid was filtered. The polymer was further purified by Soxhlet extraction using methanol, hexane, and chloroform. The chloroform fraction, precipitated in methanol gave a blue polymer. The molecular mass of this batch was estimated by SEC as $\mathrm{M}_{\mathrm{n}}=7.35 \mathrm{kDa}, \mathrm{M}_{\mathrm{w}}=14.3 \mathrm{kDa}$ and $\mathrm{PDI}=1.9$.

Poly[[2,5-di(2-octyldodecyl)pyrrolo[3,4-c]-pyrrole-1,4(2H,5H)-dione-3,6-diyl]-alt-[2,2':5',2':5',,

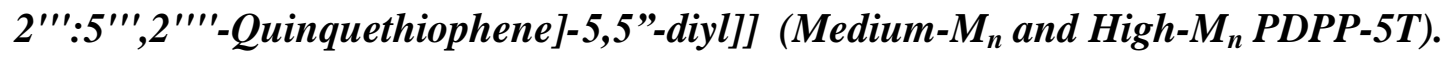

The polymer was synthesized according to the literature [4]. M1 (300 mg, 0,29 mmol, 1eq.), M2 (166 mg, 0,29 mmol, 1eq.) were dissolved in $7 \mathrm{~mL}$ of anhydrous chlorobenzene and $0.7 \mathrm{ml}$ of anhydrous DMF. Then, tris(dibenzylideneacetene)dipalladium(0) $(8 \mathrm{mg}, 8.7 \mu \mathrm{mol}, 0.03 \mathrm{eq}$.$) and$ triphenylphosphine $\left(10.4 \mathrm{mg}, 34 \mu \mathrm{mol}, 0.118\right.$ eq.) were added. The mixture was stirred at $120^{\circ} \mathrm{C}$ for $48 \mathrm{~h}$ followed by addition of the end-cappers. The mixture was stirred at $120^{\circ} \mathrm{C}$ for 6 additional hours. The mixture was precipitated in methanol and the solid was filtered. The polymer was further purified by Soxhlet extraction using methanol, hexane, chloroform and chlorobenzene. The chloroform (Medium-M $\boldsymbol{M}_{n}$ PDPP-5T) and chlorobenzene (High-M $\boldsymbol{M}_{n}$ PDPP-5T) fractions, precipitated in methanol, gave dark blue polymers. The molecular masses of Medium- $M_{n}$ and High- 
$M_{n}$ samples were estimated by SEC respectively as: Medium- $M_{n}$ PDPP-5T $\left(M_{n}=11.2 \mathrm{kDa}, M_{w}=\right.$ $50.0 \mathrm{kDa}(\mathrm{PDI}=4.4))$ and high-M $\mathrm{M}_{\mathrm{n}} \mathrm{PDPP}-5 \mathrm{~T}\left(\mathrm{M}_{\mathrm{n}}=16.9 \mathrm{kDa}, \mathrm{M}_{\mathrm{w}}=46.3 \mathrm{kDa}(\mathrm{PDI}=2.7)\right)$.

\section{REFERENCES}

[1] N. Berton, C. Ottone, V. Labet, R. de Bettignies, S. Bailly, A. Grand, C. Morell, S. Sadki, F. Chandezon, Macromolecular Chemistry and Physics 2011, 212, 2127.

[2] L. Huo, J. Hou, H.-Y. Chen, S. Zhang, Y. Jiang, T. L. Chen, Y. Yang, Macromolecules 2009, 42, 6564 .

[3] B. S. Souza, E. C. Leopoldino, D. W. Tondo, J. Dupont, and F. Nome, Langmuir 2012, 28, 833-840.

[4] Y. Li, S. P. Singh and P. Sonar, Adv. Mater. 2010, vol. 22, no. 43, 4862-4866.

[5] E. Zhou, Q. Wei, S. Yamakawa, Y. Zhang, K. Tajima, C. Yang, K. Hashimoto, Macromolecules 2010, 43, 821.

[6] R. Di Pietro, T. Erdmann, J. H. Carpenter, N. Wang, R. R. Shivhare, P. Formanek, C. Heintze, B. Voit, D. Neher, H. Ade, A. Kiriy, Chem. Mater. 2017, 29, 10220.

[7] Y. Ji, C. Xiao, Q. Wang, J. Zhang, C. Li, Y. Wu, Z. Wei, X. Zhan, W. Hu, Z. Wang, R. A. J. Janssen, W. Li, Advanced Materials 2016, 28, 943. 